\title{
A Systematic Review of Finger Vein Recognition Techniques
}

\author{
Kashif Shaheed ${ }^{1}\left(\mathbb{D}\right.$, Hangang Liu ${ }^{2}$, Gongping Yang ${ }^{1, *}, \operatorname{Imran}$ Qureshi ${ }^{1}$, Jie Gou ${ }^{1}$ and \\ Yilong Yin ${ }^{1}$ \\ 1 School of Computer Science and Technology, Shandong University, Jinan 250101, China; \\ kashifshaheed1@gmail.com (K.S.); imarwat11@gmail.com (I.Q.); 123456.xiaojie@163.com (J.G.); \\ ylyin@sdu.edu.cn (Y.Y.) \\ 2 Shandong Province Water Conservancy Information Center, Jinan 250002,China; liu_hangang@163.com \\ * Correspondence: gpyang@sdu.edu.cn; Tel.: +86-531-8839-2498
}

Received: 26 July 2018; Accepted: 14 August 2018; Published: 24 August 2018

\begin{abstract}
Biometric identification is the study of physiological and behavioral attributes of an individual to overcome security problems. Finger vein recognition is a biometric technique used to analyze finger vein patterns of persons for proper authentication. This paper presents a detailed review on finger vein recognition algorithms. Such tools include image acquisition, preprocessing, feature extraction and matching methods to extract and analyze object patterns. In addition, we list some novel findings after the critical comparative analysis of the highlighted techniques. The comparative studies indicate that the accuracy of finger vein identification methods is up to the mark.
\end{abstract}

Keywords: biometrics; finger vein recognition; feature extraction; matching; performance analysis

\section{Introduction}

The ability to identify individual attributes in the smart recognition field is a global security concern [1]. In recent years, various algorithms have been developed to address the security problem, but there is still room for fast and efficient biometric recognition. Biometric recognition refers to an automatic recognition of individual properties acquired by their anatomic/behavioral characteristics. Several types of biometric techniques have been presented based on these anatomic/behavioral features such as fingerprint, palm print, hand veins, finger veins, palm veins, foot vein, iris, gait, DNA recognition, palates, voice recognition, facial expression, heartbeat, signature, body language, and face shape [2]. These biometric recognition approaches can be divided into two categories: (i) extrinsic biometric features (palm print, iris, fingerprint, face) and (ii) intrinsic biometric features (palm vein, hand vein, and finger vein) [3]. Extrinsic features are more visible and have more adverse factors as compared to the intrinsic features. For instance, the retinal surface is affected by the high intensity of light during extraction of iris features [4]. Similarly, the accuracy of face identification is also distorted due to brightness variance, style of facial, blockage of blood vein and pose [5]. Table 1 present the merits, defects and other characteristics of some typical extrinsic and intrinsic features.

Table 1. Characteristics of typical extrinsic and intrinsic features.

\begin{tabular}{cccccc}
\hline Biometric Technique & Level of Security & Major Advantage & Disadvantage & Cost & Sensor \\
\hline Face & Normal & Remote capture & Lighting conditions & Low & Non-contact \\
Voice & Normal & Natural and convenient & Noise & Low & Non-contact \\
Fingerprint & Good & Widely applied & Skin & Low & Contact \\
Iris & Excellent & High accuracy & Glasses & High & Non-contact \\
Finger vein & Excellent & High security level & Disease & Low & Non-contact \\
\hline
\end{tabular}


In 2002, Kono [6] —a Japanese medical researcher-introduced finger vein recognition techniques. Since then, these techniques have been widely implemented in hundreds of cities in Japan, and other countries worldwide have developed finger vein identification systems [7]. The vein-based authentication system contains biometric pattern models for security and convenience for personal identification. The typical framework of the biometric finger vein recognition (FVR) system is presented in Figure 1. The vein is a part of intrinsic features, and is therefore difficult to duplicate and falsify. Finger veins are frequently captured using near-infrared (NIR) light (700-900 nm) in a trans-illumination manner [8]. Vein-based systems usually utilize various anatomic features like finger vein, hand vein, foot vein or palm vein for personal verification. Finger vein is preferable in that its imaging tool is smallest, and the fact that fingers have a larger number of veins than the palm and hand [5]. In addition, a every finger vein pattern is unique even for identical twins and exists only for live humans [9]. Most importantly, each finger vein pattern does not change during a lifetime [4].

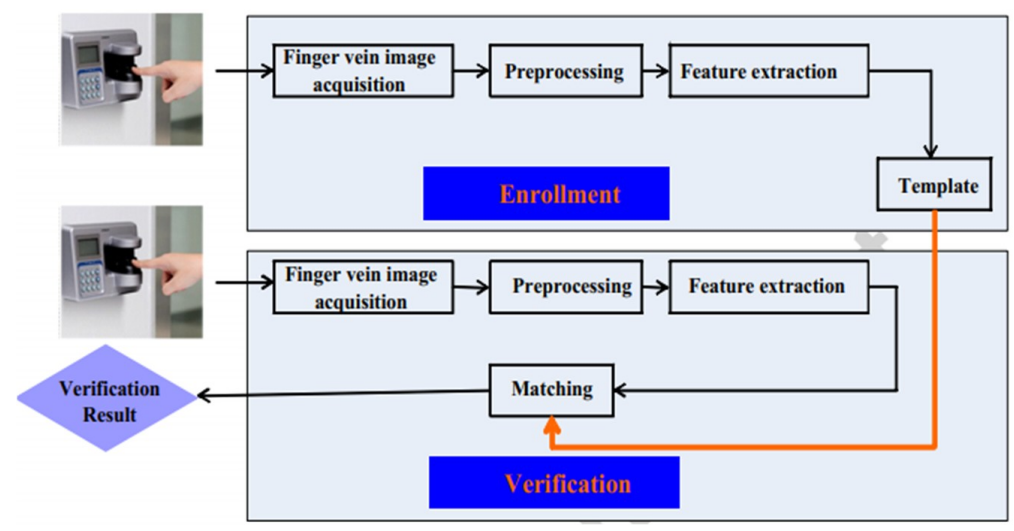

Figure 1. Typical framework for finger vein recognition [10].

It is considered that finger vein recognition is a challenging task because of low image contrast, uneven-illumination and temperature variations. Finger vein identification systems are also vulnerable to spoof attacks $[4,8,11,12]$; however, accuracy of personal verification is the most serious issue. Therefore, fast and efficient methods are still required for FVR.

The main objective of this study is to analyze the most recent techniques applied in finger vein identification. The rest of the paper is arranged as follows: Section 2 discusses various publicly available material commonly used in the finger vein identification systems. Section 3 highlights in detail existing finger vein recognition techniques, Section 4 provides the objective comparison of the reviewed conventional, machine learning and deep learning approaches and Section 5 discusses the prospects for finger vein systems. The conclusion is presented in Section 6.

\section{Materials}

There are many open finger vein databases such as SDUMLA-HMT [13], HKPU-FV [14], UTFV [15], MMCBNU_6000 [16], THU-FV [17], FV-USM [18] University developed their finger vein database called (HKPU-FV) [14], which consists of finger vein and low texture images. In 2010, Shandong University released one multimodal trait database SDUMLA-FV [13]. The third database UTFV [15] is presented by University of Twente. In the recent past, two finger vein databases, THU-FV [17] and MMCBU_6000 [16], were published by Tsinghua and Chunbuk Nation University respectively. All these public databases provide more than 100 subjects of finger veins, except UTFV database which provides 60 subjects. FV-USM was the infrared finger vein image dataset which was developed by University Sains Malaysia in 2013 [19]. In 2014, the VERA database was produced by the Idiap Research Institute in Martigny and Haute Ecole Specialisee de Suisse Occidentale in Sion, in Switzerland [20]. Some of the aforementioned databases may be well suited for one specific application and may not suit other applications. Table 2 illustrate the finger vein datasets. 
Table 2. Detail of finger vein image datasets.

\begin{tabular}{|c|c|c|c|c|c|c|c|}
\hline Database & $\begin{array}{l}\text { Acquisition } \\
\text { Method }\end{array}$ & $\begin{array}{c}\text { No. of } \\
\text { Subjects }\end{array}$ & No. of Images & No. of Fingers for Each Subject & $\begin{array}{l}\text { No. of Images } \\
\text { for Each Subject }\end{array}$ & Image Format & Size of Images \\
\hline SDUMLA-FV [13] & Light transmission & 106 & 3816 & 6 (both hands middle, index, ring) & 6 & bitmap & $320 \times 240 \mathrm{pxl}$ \\
\hline THU-FV [17] & Light transmission & 220 & 440 & 1 & 1 & bitmap & $200 \times 100 \mathrm{pxl}$ \\
\hline UTFV [15] & Light transmission & 60 & 1440 & 6 (both hands middle, ring, index) & 4 & $\begin{array}{c}\text { PNG, } 8 \text { Bit Gray } \\
\text { Scale }\end{array}$ & $672 \times 380$ pxl \\
\hline HKPU-FV [14] & Light transmission & 156 & 6264 & 3 (left hand middle, ring, index) & $12 / 6^{*}$ & bitmap & $513 \times 256 \mathrm{pxl}$ \\
\hline MMCBNU_6000 [16] & Light transmission & 100 & 6000 & 6 (both hands middle, ring, index) & 10 & bitmap & $640 \times 480 \mathrm{pxl}$ \\
\hline FV-USM [18] & Light transmission & 123 & 5904 & 4 (both index fingers, both middle fingers) & 6 & bitmap & $640 \times 480 \mathrm{pxl}$ \\
\hline VERA [21] & Light transmission & 110 & 440 & 2 (left index and right index) & 2 & PNG & $665 \times 250 \mathrm{pxl}$ \\
\hline
\end{tabular}

* For the second imaging session, there were only 105 subjects turned up, so each of finger from these subjects has 6 images, but others each has 12 images. 


\section{Finger Vein Recognition (FVR)}

Generally, FVR consists of image acquisition, preprocessing, feature extraction and matching methods. This section presents a detailed discussion on finger vein identification approaches related to the steps mentioned above. For ease of comprehension, the block diagram of the related literature is shown in Figure 2.

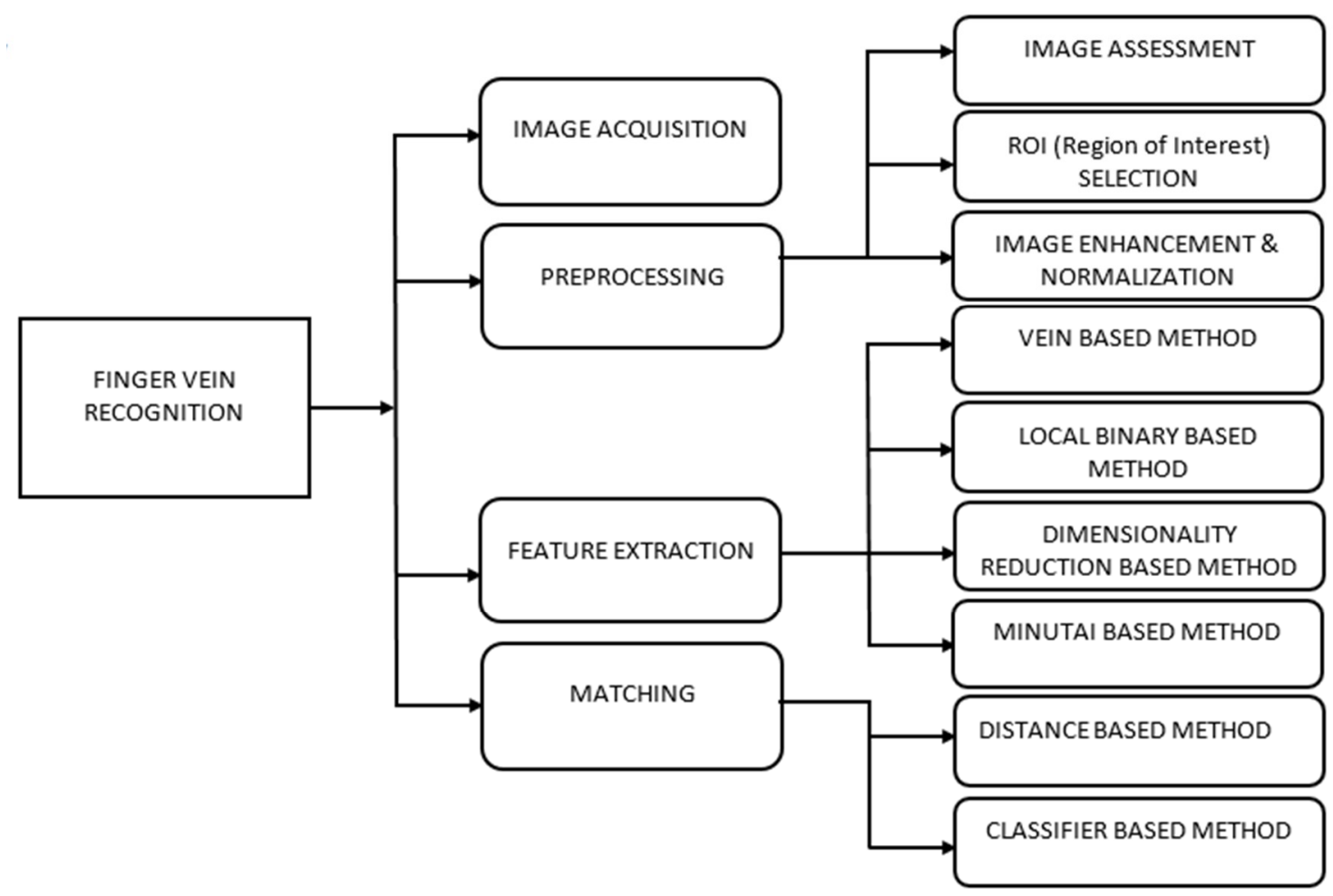

Figure 2. Block diagram of finger vein recognition techniques.

\subsection{Image Acquisition}

Image acquisition is the first basic step in FVR in which the finger vein image is captured by using NIR (near infra-red) light in the illumination transaction method. The acquisition device consists of an NIR assembly part for placement of the finger, and a charge-coupled device (CCD) preprocessor camera is then used to obtain an image of the finger vein [22,23]. The NIR light can pass through a finger but hemoglobin in the blood can absorb more NIR light than other tissues (such as bones and muscles) [9]. When the vein of a finger absorbs infrared light, the image of the finger vein can be acquired as a dark line. Figure 3 shows how the finger vein scanner works, and Figure 4 presents the finger vein scanner. NIR imaging is secure because it passes through the finger to capture the images [20]. Three methods are mainly used for finger vein image acquisition: light transmission method, light reflection method and two-way radiating method [24,25]. Among these methods, a high contrast image is captured using the transmission method, therefore most of finger vein imaging devices employ the light transmission method [26]. However, for optimization of the image acquisition device, there are always some problems, such as low contrast, translational and rotational variation and noise, which cannot be solved during the image capturing process, hence the next step-image preprocessing - which resolves these problems. 


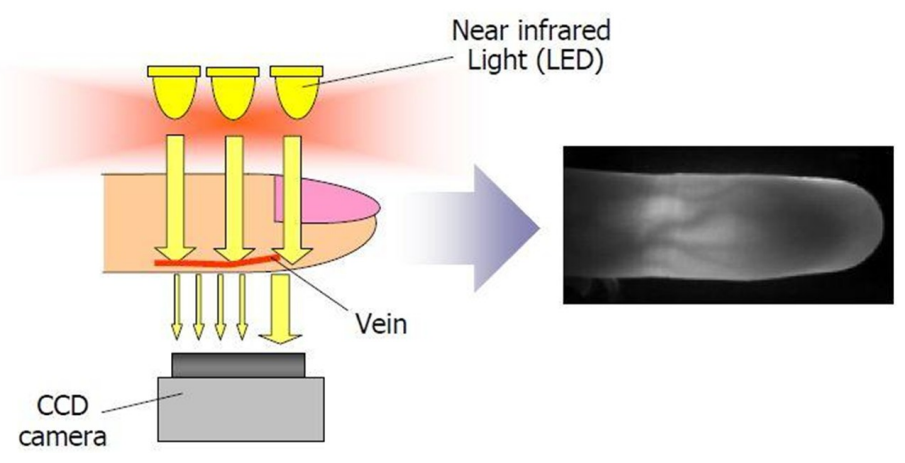

Figure 3. How a finger vein scanner works [27].

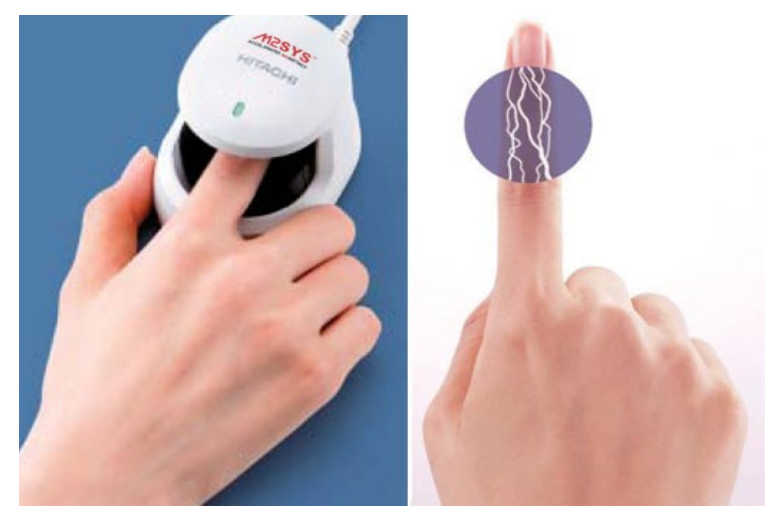

Figure 4. A finger vein scanner [28].

\subsection{Preprocessing}

Before the feature extraction step, the data from the image sensor device should be preprocessed. The objective of image preprocessing is to provide a robust Region of Interest (ROI) image for feature extraction. Good performance of a finger vein image depends on the finger vein image quality [29]. The finger vein image usually consists of noise, shades and low contrast. This is because of light fluctuation, a rotational and translational variation of the finger and also the performance of the capturing device. The preprocessing step is applied to relieve these problems. The three common preprocessing stages are (i) image quality assessment, (ii) region of interest (ROI) extraction, and (iii) normalization and enhancement.

\subsubsection{Image Quality Assessment}

Image assessment acts as the first sub-step of preprocessing. During this stage, quality of acquired image samples is examined to estimate the suitability for further processing. If the raw data of the required sample is not satisfactory then there are two options. One is to acquire the data again from the user and another option is to generate an exception which alerts the administrator to implement other suitable procedures [1]. Recently, several quality assessment schemes have been proposed to advance the performance of finger vein identification. To evaluate the quality of images, HSNR (signal to noise ratio based on the human visual system) evaluation index is proposed to simulate the properties of the human visual system [30]. Based on Radon transform, Qin et al. [31] proposed a new image quality assessment algorithm to enhance the performance of finger vein system, where the quality score was predicted from the curvature of corresponding Radon Space. A quality metric finger vein quality evaluation based on a hierarchical feature of the vein was presented, which was shown with better matching performance [32]. In order to reduce the authentication error rate, Nguyen et al. [33] measured the finger vein image quality by detecting number of vein points in the 
image. Yang et al. [13] estimated the quality of finger vein by combining image contrast, information capacity, and gradient, based on SVM, and filtered out the low-quality images. Peng et al. [34] used a triangular norm approach for performance improvement using attributes illustrated in Reference [35]. Zhou et al. [36] associated three local and two global level features based on SVR to determine the quality of the finger vein image, but the scores of training data need to be annotated manually in advance. To predict vein quality and also to reduce recognition error rate, Qin et al. [37] proposed a Deep Neural Network (DNN) for representation learning from the binary image, although it does not rely upon mean, energy and contrast features of the finger vein image; however it is complex in its application and requires more training data to train the deep neural network. Extracted stable and prominent features from a finger vein image can obviously upgrade the quality assessment of a vein image. Huang et al. [38] proposed a method based on information fusion of structure and texture that takes both the recognition strategy and feature extraction into account, which in turn enhances the recognition performance.

\subsubsection{ROI Extraction}

The second most important stage is ROI extraction. In finger vein images, there are undesirable regions (image background) and the valuable area (finger area) in the image. The valuable area is called ROI, and ROI extraction is the processing to localize and extract the finger area from the captured image and delete the image background [39]. Different approaches are used to segment the finger region from the captured image such as region-based method, thresholding, template method, and edge-based method [40]. Brindha et al. [39] extracted the ROI from the original image by using two morphological operations. Wang et al. [29] extracted the ROI from finger vein image by cutting the maximum inscribed rectangle of the finger vein image, and the original image was binarized using a selected threshold [29]. In Reference [41] the ROI was obtained from the original image by using Sobel operator. In References [42,43], the finger region was segmented by using sub window scheme.

Multiple image acquisitions are used in finger vein recognition system. Hence, different imaging devices using the ROI extraction method face challenges, such as gray level, image size variation, and background noise appearances in finger vein images, which affect the performance of the ROI extraction method. In order to address the sensor impact on finger vein images in a finger vein recognition system, Yang et al. [44] proposed a cross-sensor super-pixel-based ROI extraction method. In this work, the super-pixel segmentation method was conducted on the finger vein image and finger vein boundaries were tracked from the segmented image. One other issue in former ROI extraction approaches is that some important information is lost in cropped ROI. In order to solve the information loss problem, Wang [45] proposed a new ROI extraction method for finger vein images with fewer information losses by using modified sliding window method and outer rectangle method. The parameter used in the algorithm is also very important and they will influence the performance of the finger vein recognition systems. Zhang et al. [46] presented a parameter adjustment method in the preprocessing stage to select the filter in noise reduction and threshold in edge detection.

\subsubsection{Normalization and Enhancement}

Normalization is a process that normalizes the range of pixel intensity values in an image. After extracting the ROI, the finger vein image is normalized in order to accommodate geometric changes and to get consistent image size [47]. In addition, normalization in the preprocessing stage eliminates the diverse variation problems of the image [48].

Image enhancement is another key stage in the preprocessing phase. The basic goal of image enhancement is to advance the interpretable or knowledge of information in images for human viewers or to get the standard enhanced image from the unclear acquired image [29]. In finger vein recognition, image enhancement is required to get better matching performance. Enhancement of a finger vein image mainly focuses on contrast enhancement and noise removal. There are many enhancement techniques used to improve the image quality. Contrast limited adaptive 
histogram equalization (CLAHE) is one of the common enhancement approaches in finger vein recognition [48,49]. Additionally, some other enhancement algorithms have also presented good results [41,49-51]. Yang et al. [52] have employed Circular Gabor Filter (CGF) approach to improve finger vein images. Histogram equalization technique was employed by Liu and Song to enhance the gray level contrast in finger vein images [24]. Gaussian matching technique was proposed by Wang [29] to enhance finger vein image. Adaptive histogram equalization enhancement was implemented by Reference [10] to detect edges of vein significantly and enhanced the global contrast of input image. In References [5,25,31,48,53], Gabor filter technique was used to enhance finger vein images and remove noise from images. Zhang et al. applied CGF for image enhancement of finger vein [54]. Xie et al. proposed a normalization method for finger vein image enhancement using a guided filter-based single scale retinex (GFSSR) [48]. Moreover, some enhancement techniques were combined to enhance the finger vein image and reduce the noise. Pflug et al. employed adaptive non-local mean and non-linear diffusion method to enhance image and reduce noise [55]. Kayode et al. [19] combined Gabor filter and canny edge detector to enhance the finger vein image and remove noise.

\subsection{Feature Extraction}

Feature extraction represents one of the most crucial and major steps of FVR. During this step, the quantifiable property of the basic biometric trait is created, called the template, which is helpful for identifying the individual. For example, in a fingerprint biometric system [1], position and orientation of minutiae points in a fingerprint image is the key feature which needs to be different from another person. An efficient feature extraction technique is a step which enhances the precision of finger vein recognition. Numerous feature extraction techniques have been presented, and this paper discusses four groups of feature extraction methods, i.e., vein-based method, local binary-based method, dimensionality-based method and minutiae-based method.

\subsubsection{Vein-Based Method}

Gabor filtering is an impressive feature extraction technique in snatching texture characteristics from an image and is therefore employed in various pattern recognition applications i.e., finger print identification, hand vein identification and iris identification [47]. Gabor filtering is also valuable for finger vein extraction due to its directional acuteness, detecting oriented feature capability and fine tuning to a specific frequency. The General form of Gabor filtering can be represented as:

$$
(x, y, f, \varphi)=\frac{1}{2 \pi \sigma_{x} \sigma_{y}} \exp \left[-\frac{1}{2}\left(\frac{x_{\varphi^{2}}}{\sigma_{x^{2}}}+\frac{y_{\varphi^{2}}}{\sigma_{y^{2}}}\right)\right] \cos \left(2 \pi f x_{\varphi}\right)
$$

Here, $x, y$ represent the image $x_{\varphi}$ and $y_{\varphi}$ where $\varphi$ represent the orientation and $f$ show the frequency of a sinusoidal plane.

There are some Gabor filter-based FVR techniques. Kumar et al. [14] used multi-orientation for finger vein pattern extraction. Yang et al. proposed multi-channel Gabor filter and a bank of even-symmetric Gabor filter with eight orientations to get information about vein vessel [56,57]. In References [50,58], 2D Gabor filter was applied to extract direction texture and phase feature from finger vein image, and good recognition results were obtained. Xie et al. proposed guided Gabor filter method which obtained vein pattern without involving any segmentation process [59]. Multi-orientation Gabor filter was used for FVR in Reference [60]. Sapkale et al. [22], presented fractal dimension, lacunae extraction and Gabor filter algorithm for texture and edge feature extraction. In Reference [61], Gabor filter was applied to choose the characteristics texture extraction of vein images that capture the local orientation and the information of the frequencies of the vein network.

Repeated line tracking is also a vein pattern extraction technique that tracks vein patterns a particular number of times. Line tracking operation recognizes the local dark line (finger vein pattern) and executes pixel by pixel along black lines. If a black line is not detected, the new tracking point 
starts randomly at another position. In such a way, repeated line tracking operation tracks all the black lines in the image. Finally, a vein pattern of the finger is obtained. The repeated line tracking method has been proposed by Miura et al. [9] to obtain the finger vein pattern from an ambiguous image. However, the algorithm proposed in Reference [9] contains some drawbacks such as low robustness and complexity. Robustness and efficiency of the repeated line tracking algorithm relate to parameter $p_{n 0}$ described in Reference [62], $p_{n 0}$ is the point used to start the line tracking, to select the useful point for line tracking, and revise the parameter for finger vein image according to the width of vein for different images. To evaluate the locus space of finger vein, Liu et al. [62] presented a modified repeated line tracking (MRLT) on revised parameters which proved to be effective. However, such a proposed method lacks metric quantitative evaluation for image segmentation. To get a good result, the repeated line tracking method was combined with Gabor filter to extract features $[63,64]$. Repeated line tracking and Gabor filter methods were used to attain features from finger vein image and obtained a better result as compared to the separate result of both these techniques. Nonetheless, these algorithms are time consuming and complex. In multimodal biometric recognition system, repeated line tracking technique was also implemented to obtain feature extraction. In Reference [65], the repeated line tracking approach with feature-level fusion using fractional firefly (FFF) optimization was employed to extract features from finger knuckle and finger vein images.

Maximum curvature method is another excellent method to extract finger vein patterns. It utilizes the fact that vein patterns appear like a valley with high curvature in the cross-sectional profile. The curvature of finger vein is computed and only the centerlines of veins are saved. The centerlines are detected by searching for positions which have the locally maximal curvatures of a cross-sectional profile. Miura et al. proposed to use local maximum curvatures in cross-sectional profiles to extract vein pattern from the images with various widths and brightnesses [66].

The mathematical form of curvature, $k(z)$ as follows:

$$
K(z)=\frac{d^{2} P f(z) / d z^{2}}{\left\{1+(d P f(z) / d z)^{2}\right\}^{\frac{3}{2}}}
$$

$P f(z)$ represents a cross-sectional profile acquired from an image at any position and direction. The profile of $k(z)$ is classified into convex and concave. The local maximum of $k(z)$ is calculated at each concave area indicating center points of the veins. Score is calculated for each center position and all four direction are analyzed, and finally the image is obtained by selecting the maximum curvature points.

Tagkalakis et al. [67] also use maximum curvature point to obtain features from a captured finger vein image. However, the vein pattern produced in References [66,67] are uneven due to the fact that maximum curvature is estimated in only four directions (two diagonal, vertical and horizontal). Song et al. [68] proposed a mean curvature method which used geometrical properties of the intensity field to obtain vein pattern from unclear vein images in all direction. However, during the matching stage of algorithm, matched pixel ratio method was employed for binarized patterns that required whole finger vein pattern, which can expose the system to attacks on personal information. In finger vein verification, extracting a finger vein pattern from the infrared image is difficult. Choi et al. [69] proposed a feature extraction algorithm employing gradient normalization and principal curvature methods and obtained better performance than References [66,68]. In order to systematically differentiate between the individual finger vein samples, Yahya et al. [70] proposed maximum curvature and directional based features (MCDF) and discretization method to improve identification performance.

\subsubsection{Local Binary-Based (LBP) Method}

Local Binary Pattern is a local feature descriptor used to represent the finger vein local feature information. The LBP code may be described as an ordered set of binary values determined by 
comparing the gray value of a central pixel with its neighboring pixels. In Reference [71], the ordered set of binary values can be represented in a decimal form as shown below:

$$
\operatorname{LBP}\left(x_{c}, y_{c}\right)=\sum_{n=0}^{7} S\left(i_{n}-i_{c}\right) 2^{n}
$$

where, $i_{n}, i_{c}$ denotes the gray value of the central pixel $\left(x_{c}, y_{c}\right)$ and the gray values of the eight surrounding pixels, respectively.

Several approaches have been developed to extract a local feature of finger vein images. For example, Lee [71-73] used the locally based feature extraction scheme to remove the problem of irregular shading and the highly saturated area in the image, which greatly reduced the processing time and improved recognition. Rosdi et al. [74] introduced the local line binary pattern (LLBP) approach to extract features and achieved excellent results over previous methods [71,75]. LLPB method is a revised version of LBP (Local binary pattern), which extracts feature in both horizontal and vertical direction. Finger vein images have rich orientation information, and the line patterns obtained only from vertical and horizontal orientation may not have enough discrimination information for matching. To further enhance the discriminatory information, $\mathrm{Yu}$ et al. proposed polydirectional line pattern (PLLBP) [76] and generalized local line binary pattern (GLLBP) [77] methods, which extract line pattern at an arbitrary orientation. However, LLBP and PLLBP have low discriminatory information and a jumble of redundant information. Hence, Liu et al. advance a novel customized local line binary pattern (CLLBP) [78] approach to eliminate the information reduction, increase discriminatory information of local features and reduce the matching time of recognition system. Extraction of powerful feature greatly improves the performance of finger vein identification. For extracting powerful features, $\mathrm{Xi}$ et al. [79] introduced Pyramid histograms of gray, textures and orientation gradient (PFS-PHGTOG) technique by discriminating subset features from PHGTOG to reduce the verification error rate (EER). Translation and rotational variation of finger cause change of finger vein image which degrades performance of the FVR system. To deal with this difficulty, Wang [29] and Yang [80] proposed translational and rotational invariant feature extraction approach for FVR. A remarkable result is obtained in Reference [81]. A Multi-scale Sobel Angle Local Binary Pattern (MSALBP) novel feature extraction approach is presented for personal verification using finger texture pattern of five fingers. $\mathrm{Xi}$ [79] proposed feature extraction technique which outperforms the existing techniques. References $[10,82]$ are finger vein identification technique which also used the local binary feature.

\subsubsection{Dimensionality Reduction-Based Method}

In dimensionality reduction-based method, finger vein image is transformed into a low dimensional space by dimension reduction, in which the discriminating information is kept and noises are discarded. Mostly feature extraction technique in this kind of method requires a training process to learn the transformation matrix, and a classifier is employed in the matching process. During image acquisition, the residual information is also obtained such as pose variation, shades of finger muscle and bone around the vein, which may affect the accuracy of the identification system. In order to remove these problems, Liu et al. [83] presented Orthogonal Neighborhood Preserving Projection (ONPP) manifold learning method for the first time to handle the pose variation problem in finger vein image and obtained $97.8 \%$ recognition rate. Due to the acquisition devices, torsion, translational and other deformation in the local area, the traditional approach achieved very low identification precision. Guan et al. [84] proposed a feature extraction method named Bi-directional Weighted Modular B2DPCA (BWMB2DPCA), which obtained a better result than the traditional techniques. The BWMB2DPCA method shortened the size of image feature matrix, which also decreased the recognition accuracy as different feature vectors effect identification efficiency. Additionally, the experimental result of BWMB2DPCA was not conclusive because the eigenvectors of column are ignored. 
Therefore, method B2DPCA (BWMB2DPCA) in Reference [84] was proposed to the feature matrix $\mathrm{Z}={ }^{T}$ as follows

$$
\left\{\begin{array}{c}
Z=B_{W}^{T} A X_{w} \\
Z=(B \times \operatorname{diag}(\lambda \times 1, \lambda \times 2, \lambda \times 3, \ldots, \lambda \times d))^{T} A(X \times \operatorname{diag}(\lambda \times 1, \lambda \times 2, \lambda \times 3, \ldots, \lambda \times d))
\end{array}\right.
$$

where $w$ shows the weighted factor, $\lambda$ represent the separate values of column or row directions. The eigenvalue normalized formula is as follows

$$
\lambda_{i}^{\prime}=\frac{\lambda_{i}-\lambda_{\min }}{\lambda_{\max }-\lambda_{\min }} i=1,2, \ldots, t, \ldots d
$$

where $\lambda_{\max }$ and $\lambda_{\min }$ represent the maximum and minimum eigenvalue of the column and row direction.

However, B2DPCA in Reference [84] cannot extract the best discriminative features. Wu in $[85,86]$ presented the PCA method with different machine learning classifier which extracted the most distinguished feature. Furthermore, the method in References $[85,86]$ greatly reduces the computational cost, removes noise and enhances identification accuracy. Yang et al. [87] proposed (2D) ${ }^{2}$ PCA method for feature extraction, which is more effective than the method in Reference [86] and B2DPCA. The dimension of the feature is considered very important; the system consumes more time if the dimension of the feature vector is high. However, if the dimension of the feature vector is low, the system might ignore some useful information. To resolve this issue, Haijian et al. [88] used optimal dimensional kernel feature reduction technique (KPCA) to keep the balance between accuracy and speed. In addition, due to some factors such as horizontal displacement, lights, and temperature, the vein image has some degree of nonlinear distributive properties. You et al. [89] employed B2DPCA and KMMC algorithm both to extract the nonlinear feature and outperform the algorithms in References [90,91].

\subsubsection{Minutiae Point-Based Method}

The point where the ridge lines end or fork is defined as a minutiae point. Minutiae points refer to the terminal point and bifurcation point of blood vessels, and are one kind of important feature of a finger vein image. Minutiae points are used in finger vein recognition, and such methods are already used in fingerprint recognition technique [92-95]. Mantrao et al. [96] presented a minutiae-based point feature extraction and matching approach which greatly improved the performance of the identification system. Aziz et al. [97] extracted minutiae points of finger vein by combining two methods, namely maximum curvature points and fingerprint application method. Prabhakar et al. [98] used endpoints and bifurcation points to eliminate false minutiae and make the identification more precise.

\subsection{Matching}

The matching technique is the last step of recognition to decide whether an input image is genuine or an imposter for one enrolled image, in which a matching score is generated. A matching score measures the likeness between the enrolled template and the input image. Two types of matching techniques are used, i.e., distance-based matching and classifier-based matching. Conventional finger vein identification approach uses the distance-based matching technique, while by machine learning techniques finger vein recognition can employ classifier-based matching technique. Classifier-based matching techniques were employed by References $[29,49,61,65,85,86,99,100]$ for finger vein recognition systems.

Table 3 summarizes the conventional finger vein recognition approaches, and Table 4 shows the machine learning algorithms-based FVR methods. The image preprocessing methods, feature extraction methods and matching methods are all involved. 
Table 3. Traditional (conventional) method of finger vein recognition.

\begin{tabular}{|c|c|c|c|}
\hline Method of Preprocessing & Method of Feature Extraction & Method of Matching & References \\
\hline $\begin{array}{l}\text { Region of interest (ROI) extraction } \\
\text { Filters combination to remove salt-and-pepper and Gaussian noise } \\
\text { Image segmentation and denoising } \\
\text { Extraction of vein and normalization method }\end{array}$ & Two direction weighted (2D)2LDA & - & [101] \\
\hline ROI Detection image enhancement size normalization & ONPP-Manifold learning & $\begin{array}{l}\text { Manifold distance method for } \\
\text { recogntion }\end{array}$ & [83] \\
\hline $\begin{array}{l}\text { Segmentation of vein ROI } \\
\text { Interphalangeal joint prior }\end{array}$ & Steerable filter & Nearest neighbor $(\mathrm{NN})$ method & [52] \\
\hline- & BWMB2DPCA & Nearest neighbor $(\mathrm{NN})$ method & [84] \\
\hline Modified Gaussian high-pass filter & Local line binary pattern & Hamming distance & [74] \\
\hline $\begin{array}{l}\text { Elimination of background } \\
\text { Removal of noise } \\
\text { Enhancement of finger vein image } \\
\text { Brightness } \\
\text { Normalization Size }\end{array}$ & $\begin{array}{l}\text { Dynamic thresholding } \\
\text { edian filter } \\
\text { Morphological operation } \\
\text { Vein location and direction coding }\end{array}$ & Template matching & [102] \\
\hline Gaussian high-pass filter & Binarization local binary pattern & Hamming distance & [73] \\
\hline Image gray processing ROI extraction normalization & Directional Code & Matching & [103] \\
\hline $\begin{array}{l}\text { Image gray processing } \\
\text { ROI extraction } \\
\text { Normalization (size and gray) method }\end{array}$ & Personalized best bit map (PBBM) & Matching & [104] \\
\hline $\begin{array}{l}\text { Histogram equalization } \\
\text { Bucolic interpolation }\end{array}$ & $\begin{array}{l}\text { Fractal dimension } \\
\text { Wavelet transform }\end{array}$ & $\begin{array}{l}\text { Wavelet transformation } \\
\text { Energy feature }\end{array}$ & [105] \\
\hline $\begin{array}{l}\text { ROI extraction } \\
\text { CLAHE }\end{array}$ & Linear Kernel Entropy Component analysis (KECA) & Euclidian distance & [106] \\
\hline $\begin{array}{l}\text { Anisotropic diffusion method } \\
\text { Non-scatter transmission maps } \\
\text { Gabor wavelet }\end{array}$ & Directional filtering method & Phase only correlation strategy & [107] \\
\hline $\begin{array}{l}\text { ROI extraction } \\
\text { Enhancement } \\
\text { Normalization size }\end{array}$ & Local line binary pattern & PWM-LLBP & [82] \\
\hline $\begin{array}{l}\text { Edge detection } \\
\text { ROI Extraction } \\
\text { Smoothing filter }\end{array}$ & Personalized best bit map (PBBM) & Cross-correlation matching & [108] \\
\hline
\end{tabular}


Table 3. Cont.

\begin{tabular}{|c|c|c|c|}
\hline Method of Preprocessing & Method of Feature Extraction & Method of Matching & References \\
\hline ROI Extraction & (HCGR) Histogram of competitive Gabor response & Matching & [109] \\
\hline $\begin{array}{l}\text { ROI Extraction } \\
\text { Brightness normalization } \\
\text { Minimization of the Mumford-Shah Model }\end{array}$ & $\begin{array}{l}\text { Morphological dilation docal entropy thresholding } \\
\text { Morphological filtering }\end{array}$ & Template matching & [110] \\
\hline $\begin{array}{l}\text { Region of interest extraction } \\
\text { Multiscale matched filtering } \\
\text { Line tracking }\end{array}$ & Variational approach & Sum of square differences & [111] \\
\hline $\begin{array}{l}\text { ROI Extraction } \\
\text { Image enhancement }\end{array}$ & $\begin{array}{l}\text { Fractal dimension } \\
\text { Lacunae } \\
\text { Gabor filter }\end{array}$ & $\begin{array}{l}\text { Difference compared with } \\
\text { threshold value }\end{array}$ & [46] \\
\hline $\begin{array}{l}\text { Denoising } \\
\text { Image enhancement }\end{array}$ & Local binary pattern & Hamming distance & [20] \\
\hline $\begin{array}{l}\text { ROI extraction } \\
\text { Binarization } \\
\text { Thinning }\end{array}$ & $\mathrm{MCDF}$ & & [70] \\
\hline $\begin{array}{l}\text { ROI Localization } \\
\text { Image enhancement }\end{array}$ & $\begin{array}{l}\text { Uniform optimal uniform rotation invariant LBP } \\
\text { descriptor }\end{array}$ & Histogram intersection method & [112] \\
\hline $\begin{array}{l}\text { Binarized } \\
\text { ROI } \\
\text { Thinned } \\
\text { Gabor filter }\end{array}$ & Minutiae-based extraction & Euclidian distance & [24] \\
\hline $\begin{array}{l}\text { Image denoising } \\
\text { ROI localization } \\
\text { Image enhancements }\end{array}$ & $\begin{array}{l}\text { LLBP } \\
\text { PLLBP }\end{array}$ & Histogram intersection & [76] \\
\hline ROI extractions & GLLPB & Soft power metric & [77] \\
\hline $\begin{array}{l}\text { Size normalization } \\
\text { ROI Extraction } \\
\text { Gray normalization }\end{array}$ & CLLBP & Matching score & [78] \\
\hline
\end{tabular}


Table 4. Traditional Machine learning approaches for finger vein recognition.

\begin{tabular}{|c|c|c|c|}
\hline Preprocessing Method & Feature Extraction Method & Matching Method & Reference \\
\hline ROI extraction, image resize & PCA, DCA & SVM and ANFIS & [86] \\
\hline ROI extraction, image resize & PCA & ANFIS (neuro-fuzzy system) & [85] \\
\hline $\begin{array}{l}\text { ROI extraction, median filter, histogram } \\
\text { equalization }\end{array}$ & Morphological operation, maximum curvature points & MLP & [113] \\
\hline Gaussian matched filter & LBPV & Global matching, SVM & [29] \\
\hline Gabor filtering & Global thresholding, Gabor filter & SVM & [61] \\
\hline ROI extraction, normalization & $\begin{array}{l}\text { Image contrast, gradient in spatial domain, Gabor feature, } \\
\text { information capacity and entropy }\end{array}$ & SVR & [36] \\
\hline Normalization, filtering, resizing & $\begin{array}{l}\text { Grid-based location, feature-level fusion by FFF, } \\
\text { optimization }\end{array}$ & K-SVM & [65] \\
\hline
\end{tabular}




\section{Performance Analysis}

We can see the main steps of the finger vein system are stable, but they have different types of method. Performance evaluation is an important way to recognize whether these algorithms are good or bad. In this section, first the benchmarks in the evaluation of FVR performance are listed, and then the performance of various FVR techniques was inputted to a non-matching template in the dataset [114]. Receiver Operating Characteristic curve (ROC) intuitively represents the balance between False Accept Rate (FAR) and False Reject Rate (FRR). The threshold is used to make a decision on the matching algorithm. If the threshold is reduced, FAR or false match rate (FMR) increased and FRR or False Non-Match Rate (FNMR) decreased. Similarly, the higher threshold increases the FRR or FNMR and decreases the FAR or FMR. Equal error rate (EER) value can be simply attained from Receiver Operating Curve (ROC). The lower the EER, the better the system works. Fail to enroll (FTE) rate indicates the proportion of users that cannot be successfully enrolled in a finger vein recognition system, and Fail to acquire (FTA) or Fail to capture (FTC) is the error in which the finger vein biometric sensor cannot capture the sample [1].

\subsection{Conventional Finger Vein Recognition Method}

Conventional finger vein approaches were less robust to noise and misalignment than machine learning approaches; therefore, image preprocessing methods were usually applied ahead of feature extraction and matching to overcome the above-mentioned problems. Numerous conventional finger vein identification methods have been developed, but some methods $[73,76,78,83]$ have achieved remarkable development. In 2010, Lie et al. [83] proposed a finger vein verification approach, and obtained accuracy of $97.8 \%$ for identification. In term of accuracy, polydirectional local line binary pattern algorithm attained $99.21 \%$ accuracy on a dataset of 1902 images [76]. Moreover, in terms of equal error rate, conventional finger vein technique also achieved some tremendous achievements. The method proposed in References [77,78] brings about very low equal error rate of 0.61 and 0.055 on 100 and 156 subject's databases respectively. Most of the conventional finger vein recognition techniques show remarkable performance in terms of accuracy and equal error rate; however the total computational cost of the conventional finger vein algorithm is much too high $[73,74,77]$. Table 5 demonstrates the performance of typical conventional FVR techniques in aspect of accuracy, EER, FAR, FRR, Correct Classification Rate (CCR).

\subsection{Traditional Machine Learning Finger Vein Recognition Methods}

Some machine learning techniques (e.g., SVM, neural network and fuzzy logic) have been used in the feature extraction and matching stage of biometrics. These kinds of techniques have also proved to be efficient for feature extraction, matching and enhancing the performance of the FVR method. In most FVR techniques, machine learning classifier-based methods were employed during the matching stage of FVR. However, conventional finger vein approaches employ distance-based methods during the matching stage. Accuracy rate of almost all the proposed machine learning finger vein algorithms is close to $100 \%[61,85,86,105,115]$. Table 6 lists the existing literature on traditional machine learning techniques-related finger vein recognition. 
Table 5. Performance of some conventional finger vein recognition technique.

\begin{tabular}{|c|c|c|c|c|c|c|}
\hline Method & Total Images & Subject & Resolution of Image & Image Format & Performance Measure & References \\
\hline ONPP-Manifold learning & 11,480 & 164 & Not reported & Not reported & $\mathrm{EER}=0.8$ & [83] \\
\hline BWMB2DPCA & 660 & Not Reported & Not reported & Not reported & Accuracy $=97.7 \%$ & [84] \\
\hline Steerable filter & 1000 & 100 & $70 \times 170$ pixels & Not reported & $\mathrm{CCR}=98.8 \%, \mathrm{FAR}=1.32$ & [52] \\
\hline Two directional weighted (2D) $)^{2} \mathrm{LDA}$ & 660 & Not Reported & $80 \times 200$ pixels & Not reported & Accuracy $=94.69 \%$ & [101] \\
\hline $\begin{array}{l}\text { Binarization } \\
\text { Local binary pattern } \\
\text { Local derivative pattern }\end{array}$ & 2400 & 30 & $640 \times 480$ pixels & Not reported & $\begin{array}{l}\text { Binarization EER }=0.38 \text {, Processing Time }=30.6 \mathrm{~ms} \\
\mathrm{LBP} E E R=0.21,44.7 \mathrm{~ms} \text { LDP EER }=0.13,112.5 \mathrm{~ms}\end{array}$ & [73] \\
\hline Location and direction Coding (LDC) & 440 & 220 & $90 \times 40$ pixels & Not reported & $\mathrm{EER}=0.44$ & [113] \\
\hline LLBP & 2040 & 51 & $192 \times 64$ pixels & Not reported & $\mathrm{EER}=1.78$, Processing Time $=37.5 \mathrm{~ms}$ & [74] \\
\hline Linear kernal entropy Component analysis (KECA) & 2040 & 204 & Not reported & Not reported & Accuracy $=98 \%$ & [106] \\
\hline Personalized best bit map (PBBM) & 1484 & 106 & $96 \times 64$ pixels & Not reported & $\mathrm{EER}=0.0038$ & [104] \\
\hline Fractional dimension wavelet transform & 6000 & 100 & Not reported & Not reported & $\mathrm{EER}=0.07$ & [105] \\
\hline Local directional code & 4080 & 34 & $96 \times 64$ pixels & 24-bit color image & $\begin{array}{l}\text { LDC-00 }=0.0116 \\
\text { LDC- } 45=0.0102\end{array}$ & [103] \\
\hline Directional filtering method & 9000 & 100 & $100 \times 180$ pixels & 8-bit gray image & $\mathrm{EER}=0.0462$ & [116] \\
\hline Personal weight maps & 1360 & 34 & $96 \times 64$ pixels & Not reported & $\mathrm{EER}=0.0056$ & [82] \\
\hline Identification based on pattern created by finger vein & 3600 & 100 & $320 \times 240$ pixels & BMP & $\mathrm{EER}=27.56, \mathrm{GAR}=100, \mathrm{FAR}=0$ & [108] \\
\hline Histogram of competitive Gabor responses (HCGR) & 6000 & 100 & $64 \times 128$ pixels & BMP & $\mathrm{EER}=0.671$ & [109] \\
\hline PLLBP & 1902 & Not Reported & $48 \times 128$ pixels & Not reported & Accuracy $=99.21 \%$ & [76] \\
\hline GLLBP & 6000 & 100 & $64 \times 128$ pixels & BMP & EER $=0.61$, and Processing Time $=392.1 \mathrm{~ms}$ & [77] \\
\hline Variational approach & 2520 & 105 & Not reported & Not reported & EER $=4.47$ & [111] \\
\hline Maximum curvature & - & 200 & Not reported & Not reported & $\mathrm{FAR}=0, \mathrm{FRR}=1.00$ & [67] \\
\hline Spectral minutiae representation (SMR) & 5000 & 125 & Not reported & Not reported & $\mathrm{EER}=20$ & {$[50]$} \\
\hline $\begin{array}{l}\text { Radom forest regression method on efficient local } \\
\text { binary pattern }\end{array}$ & 6000 & 100 & $640 \times 480$ pixels & Not reported & $\mathrm{EER}=0.35, \mathrm{CCR}=99.65 \%$ & [99] \\
\hline Super-pixel context feature (SPCF) & $\begin{array}{c}\text { PolyU }=1872 \\
\text { SDUMLA }=636\end{array}$ & $\begin{array}{c}\text { PolyU }=156 \\
\text { SDUMLA }=106\end{array}$ & $96 \times 64$ pixels & BMP & $\begin{array}{c}\text { PolyU EER }=0.0075 \\
\text { SDUMLA EER }=0.0697\end{array}$ & [117] \\
\hline Curvature in Radon space & $\begin{aligned} \text { PolyU } & =2520 \\
\mathrm{NTU} & =680\end{aligned}$ & $\begin{aligned} \text { PolyU } & =105 \\
\text { NTU } & =85\end{aligned}$ & $186 \times 71$ pixels & Not reported & $\begin{array}{l}\text { PolyU EER }=0.48 \\
\text { NTU EER }=0.69\end{array}$ & [3] \\
\hline Scale-invariant feature transform & 2000 & 100 & $460 \times 680$ pixels & Not reported & $\mathrm{EER}=1.086$ & [118] \\
\hline CLLBP & 1872 & 156 & $96 \times 64$ pixels & Not reported & $\mathrm{EER}=0.055$ & [78] \\
\hline
\end{tabular}


Table 6. Finger vein recognition performance using traditional machine learning techniques.

\begin{tabular}{|c|c|c|c|c|c|c|}
\hline Machine Learning Approach & $\begin{array}{l}\text { Total Number of } \\
\text { Images }\end{array}$ & Subjects & Resolution of Image & Image Format & Performance Measure & Reference \\
\hline SVM & 100 & 10 & $20 \times 20$ pixels & Not reported & $\begin{array}{c}\text { Accuracy }=98.00 \% \\
\text { Processing time }=0.15 \mathrm{~s}\end{array}$ & [86] \\
\hline ANFIS (neuro-fuzzy system) & 100 & 10 & $130 \times 130$ pixels & Not reported & $\begin{array}{c}\text { Accuracy }=99.00 \% \\
\text { Processing time }=45 \mathrm{~s}\end{array}$ & [85] \\
\hline SVM & Not reported & Not reported & Not reported & Not reported & $\begin{array}{l}\text { Training data }=95.00 \% \\
\quad \text { Test data }=93.00 \%\end{array}$ & [113] \\
\hline SVM & 800 & 10 & Not reported & Not reported & $\begin{array}{c}\text { CR of Index }=90.00 \% \\
\text { CR of Ring }=96.00 \% \\
\text { CR of Middle }=90.0 \% \\
\text { CR of Little }=79.00 \% \\
\text { Index EER }=5.6 \\
\text { Ring EER }=6.5 \\
\text { Middle EER }=8.5 \\
\text { Little EER }=11.9\end{array}$ & [29] \\
\hline SVM & $\begin{array}{l}\mathrm{PKU}(\mathrm{V} 2)=200 \\
\mathrm{PKU}(\mathrm{V} 4)=160\end{array}$ & $\begin{array}{l}\mathrm{PKU}(\mathrm{V} 2)=20 \\
\mathrm{PKU}(\mathrm{V} 4)=20\end{array}$ & Not reported & Not reported & $\begin{array}{l}\text { PKU(V2) Accuracy }=98.75 \% \\
\text { PKU(V4) Accuracy }=95 \%\end{array}$ & [61] \\
\hline SVR & 1872 & 105 & Not reported & BMP & $\mathrm{EER}=4.88$ & [36] \\
\hline Multi-SVM with FFF & 1000 & 100 & $320 \times 240$ pixels & BMP & $\begin{array}{c}\text { Accuracy }=96.00 \% \\
\text { EER }=0.35, \text { FAR }=5, \text { FRR }=5 \\
\text { Processing time }=5.1 \mathrm{~s}\end{array}$ & [65] \\
\hline Multi-SVM & 612 & 17 & $320 \times 240$ pixels & BMP & $\begin{array}{c}\mathrm{EER}=0.52 \\
\text { Accuracy }=94 \%\end{array}$ & [51] \\
\hline SVM & $\begin{array}{l}\text { FV-SDU }=3816 \\
\text { FVUSM }=2952\end{array}$ & $\begin{array}{l}\mathrm{SDU}=106 \\
\mathrm{USM}=123\end{array}$ & $\begin{array}{l}\mathrm{SDU}=320 \times 240 \text { pixels } \\
\mathrm{USM}=100 \times 300 \text { pixels }\end{array}$ & BMP & $\begin{aligned} \text { FV-SDU EER } & =0.0359 \\
\text { FV-USM EER } & =0.0038\end{aligned}$ & {$[120]$} \\
\hline $\begin{array}{l}\text { Feature component-based extreme learning machines } \\
\text { (FC-ELP) }\end{array}$ & 1000 & 100 & Not reported & BMP & $\begin{array}{l}\text { Correct classification rate CCR }=99.53 \% \\
\quad \text { Computational time }=0.87 \mathrm{~ms}\end{array}$ & [115] \\
\hline
\end{tabular}




\subsection{Finger Vein Recognition Using Deep Learning Methods}

Deep Learning is a form of machine learning which include multiple layers of learning algorithms. This empowers the deep learning method to learn hierarchical representation/feature from data. Therefore, deep learning has replaced the conventional feature extraction approach in various domains involving computer vision, speech and natural language processing. Due to its strong capability with feature representation, researchers have brought deep learning in to the biometric field. In recent studies, several deep learning models are built on various datasets. However, deep learning has also been successfully applied to the field of FVR. Razdi et al. [100] used the deep learning approach for the first time in the field of FVR and accuracy of $100 \%$ was obtained on 50 subject datasets. Inspired by the recent advancement of deep learning algorithms in various research domains, Qin et al. [121] proposed a deep learning model for finger vein verification and achieved error rates of 2.70 and 1.42 on two different databases, respectively. Experimental results of Reference [121] outperform some traditional conventional finger vein recognition approaches. Moreover, Qin et al. [122] have successfully applied a deep learning algorithm to assess the quality of finger vein image and have achieved higher identification accuracy with respect to current traditional state-of-the-art image quality assessment. In Reference [123], a deep convolutional neural network (DCNN) with hard mining finger verification method was proposed which achieved better performance than commercial finger vein verification methods. This method [123] also accelerates the whole training process. The large template size requires a huge amount of storage space. Convolutional neural network (CNN) and supervised discrete hashing finger vein identification method [124] was proposed to overcome this problem, which reduce the template size by up to 250 bytes ( 2000 bits) and also increases the matching speed. Traditional finger vein identification methods are exposed to hackers because the template used by the system is in the form of bare data. Lie et al. [125] proposed a secure efficient and revocable finger vein template using deep learning and random projection, namely FVR-DLRP. Furthermore, most of the conventional finger vein recognition techniques have a misalignment and shading problem. In addition, conventional approaches require complex and complicated preprocessing and feature extraction, steps which need much more processing time and effort. To alleviate these problems, Hyung et al. [126] proposed a robust deep CNN model and an error rate of 0.396 was attained on a good quality database. Moreover, Gesi et al. [127] applied a convolutional neural network (CNN) finger vein recognition method and achieved almost 100\% accuracy, obtaining a very low error rate of 0.21 . Due to relatively small databases of finger vein, the training requirement to train deep neural network is a challenge. To address this problem, Fang et al. [128] proposed a lightweight deep learning framework, which resolved the lack of training data issue through the use of a similarity measure network. Table 7 demonstrates the performance analysis of some recent deep learning approaches in finger vein recognition. 
Table 7. Finger vein recognition performance using deep learning methods.

\begin{tabular}{|c|c|c|c|c|c|c|}
\hline Method & Total Number of Images & Subject & Resolution of Image & Image Format & Performance Measure & Reference \\
\hline Fully convolutional network (FCN) & $\begin{array}{l}\mathrm{HKPU}=2520 \\
\mathrm{USM}=5904\end{array}$ & $\begin{array}{c}\mathrm{HKPU}=105 \\
\mathrm{USM}=123\end{array}$ & $\begin{array}{l}39 \times 146 \text { pixels } \\
50 \times 150 \text { pixels }\end{array}$ & BMP & $\begin{array}{l}\text { HKPU EER }=2.70 \\
\text { USM EER }=1.42\end{array}$ & [121] \\
\hline Patch-DNN+P-SVM & $\begin{array}{l}\text { Database } A=5904 \\
\text { Database } B=2520\end{array}$ & Not reported & $\begin{array}{l}640 \times 480 \text { pixels } \\
256 \times 513 \text { pixels }\end{array}$ & BMP & $\begin{array}{c}\text { High- and low-quality image accuracy } \\
\text { on Database } A=71.01 \%, 73.57 \% \\
\text { High- and low-quality image accuracy } \\
\text { on Database B }=87.08 \%, 86.36 \%\end{array}$ & [122] \\
\hline Normalization +DCNN-HM & DS1 $=5000$ & Not reported & $384 \times 512$ pixels & BMP & $\begin{array}{c}\text { DS1 EER }=0.42 \\
\text { Total execution time }=19.27 \mathrm{~ms}\end{array}$ & [123] \\
\hline CNN with SDH & 6264 & 156 & Not reported & Not reported & $\begin{array}{c}\mathrm{EER}=0.0977 \\
\text { Reduced template Size }=250 \text { bytes }\end{array}$ & [124] \\
\hline CNN & 4800 & 64 & $376 \times 328$ pixels & Not reported & $\begin{array}{c}\text { Accuracys }=99.4 \% \\
\quad \mathrm{EER}=0.21\end{array}$ & [127] \\
\hline $\mathrm{CNN}$ & 500 & 50 & $55 \times 67$ pixels & Not reported & $\begin{array}{c}\text { Accuracy }=100.00 \% \text {, total processing } \\
\text { time }=0.15 \mathrm{~s}\end{array}$ & [100] \\
\hline DNN+P-SVM & $\begin{array}{l}\text { Database } A=2520 \\
\text { Database } B=5904\end{array}$ & $\begin{array}{l}\text { Database } A=105 \\
\text { Database } B=123\end{array}$ & $\begin{array}{l}50 \times 240 \text { pixels } \\
80 \times 240 \text { pixels }\end{array}$ & BMP & $\begin{array}{l}\text { EER of high- and low-quality image on } \\
\text { Database } A=88.99 \% \text { and } 88.18 \% \\
\text { EER of high- and low-quality image on } \\
\text { Database } B=74.98 \% \text { and } 70.07 \%\end{array}$ & [37] \\
\hline CNN (Deep learning) & Good-Quality Database $=1200$ & 20 & Not reported & BMP & On Good-Quality Database EER $=0.396$ & [126] \\
\hline Two channel network learning & $\begin{array}{l}\text { MMCBNU_6000 = } 6000 \\
\text { SDUMLA }=3816\end{array}$ & $\begin{array}{l}100 \\
106\end{array}$ & $\begin{array}{l}640 \times 480 \text { pixels } \\
64 \times 128 \text { pixels }\end{array}$ & BMP & $\begin{array}{l}\mathrm{EER}=0.10 \\
\mathrm{EER}=0.47\end{array}$ & [128] \\
\hline
\end{tabular}




\subsection{Spoofing Attack (Presentation Attack) in Finger Vein Recognition}

Biometric systems are vulnerable to different kinds of attacks that may be broadly classified into two types [129]: (1) Direct attack or spoofing or presentation attack: Biometric materials are presented directly to device (sensor) in this attack. (2) Indirect attack: Part of biometric system is attacked through malware or virus. In biometric systems, the direct attack or presentation attack is of high interest because of the security evaluation in biometric systems. Finger vein recognition previously had a good spoofing resistance as compared to other biometric modalities, but recent literature has shown that FVR devices are vulnerable to spoof attack [129,130]. In 2007, Matsumoto deceive a system using a synthetic artifact, which was the first attempt to spoof a finger vein image to the best of our knowledge [12]. If spoofing of fixed finger vein (using a printed material with registered finger vein) are applied to identify the finger vein database, then the success rate to mislead FVR system is above $80 \%$ (FAR $\geq 80 \%$ ) [25]. Nowadays, study on finger vein identification is the most popular technique. Several traditional handcrafted, machine learning algorithms are proposed to detect the presentation attack in finger vein image $[12,129,131]$. In 2013, a textured based conventional presentation attack detection (PAD) method was proposed using Fourier Spectral Energy Ratio and Discrete Wavelet Transform (FSER-DWT) [132]. However, the result was not satisfactory even on a small database of 33 subjects. In the recent past, five traditional PAD methods have been proposed at the International Conference on Biometrics (ICB) 2015 [130], including Monogenic scale space (MSS), binarized statistical image features (BSIF), residual local binary pattern (RLBP), Fourier spectral bandwidth energy (FSBE) and Weber local descriptor (LPQ-WLD). However, none of them achieved $100 \%$ detection accuracy on both cropped and full image datasets. Some other conventional PAD methods-Windowed-dynamic mode decomposition (W-DMD) [129] and Steerable Pyramids [133] - also have not obtained 100\% detection accuracy on any dataset. The reason for not achieving high accuracy is the feature extractor they used; these traditional PAD methods employ handcrafted feature extractor. To remove any limitation of feature extraction in presentation attack approaches, Nguyen et al. [131] presented transfer learning convolutional neural network (CNN) with PCA and SVM. The proposed method achieved $100 \%$ detection accuracy on two large dataset Istituto Dalle Molle di Intelligenza Artificiale Percettiva (IDIAP) [130] and ISPR [132] including both cropped and full versions of images. To the best of our knowledge, it was the first attempt to detect a presentation attack using a deep learning framework. Moreover, Raghavendra et al. [134] also employed transferable deep convolutional neural network to PAD technique and obtained low attack presentation classification error rate (APCER) of $0.4 \%$ and bona fide presentation classification error rate (BPCER) of $0 \%$. Traditional handcrafted methods are not robust enough to detect presentation attacks in finger vein recognition systems because of the high computational cost, large processing time and use of small databases in those conventional methods. In addition, the detection accuracy is also limited due to the use of handcraft feature extractor. However, a spoofing countermeasure using deep learning method can greatly enhance the performance of the PAD algorithm. Moreover, with a deep learning framework we can process a huge amount of data with lower computation cost. Below, Table 8 summarizes the proposed PAD methods. 
Table 8. Existing spoofing attack methods.

\begin{tabular}{|c|c|c|c|c|c|c|}
\hline Methods & Total Number of Images & Subject & Resolution of Image & Image Format & Performance Measure & References \\
\hline FSER-DWT & $\begin{array}{l}\text { Live images }=3330 \\
\text { Fake images }=2520\end{array}$ & 33 & $640 \times 480$ pixels & Not reported & $\mathrm{EER}=1.476$ & [132] \\
\hline MSS & $\begin{array}{l}\text { Idiap database total images } \\
\text { including real and fake }=880\end{array}$ & 110 & $\begin{array}{l}\text { Full image }=665 \times 250 \text { pixels, } \\
\text { Croppped image }=565 \times 150\end{array}$ & PNG & $\begin{array}{l}\text { Half Total Error Rate (HTER) on full image and } \\
\text { cropped image }=0.00 \% \text { and } 1.25 \%\end{array}$ & [130] \\
\hline BSIF & $\begin{array}{l}\text { Idiap database total images } \\
\text { including real and fake }=880\end{array}$ & 110 & $\begin{array}{l}\text { Full image }=665 \times 250 \text { pixels, } \\
\text { Croppped image }=565 \times 150\end{array}$ & PNG & $\begin{array}{l}\text { HTER on full image and cropped image }=4.00 \% \\
\text { and } 2.75 \%\end{array}$ & [130] \\
\hline RLBP & $\begin{array}{l}\text { Idiap database total images } \\
\text { including real and fake }=880\end{array}$ & 110 & $\begin{array}{l}\text { Full image }=665 \times 250 \text { pixels, } \\
\text { Croppped image }=565 \times 150\end{array}$ & & HTER on cropped image $=0.00 \%$ & [130] \\
\hline FSBE & $\begin{array}{l}\text { Idiap database total images } \\
\text { including real and fake }=880\end{array}$ & 110 & $\begin{array}{l}\text { Full image }=665 \times 250 \text { pixels, } \\
\text { Croppped image }=565 \times 150\end{array}$ & PNG & $\begin{array}{l}\text { HTER on full image and cropped image }=0.00 \% \\
\text { and } 20.50 \%\end{array}$ & [130] \\
\hline LPQ-WLD & $\begin{array}{l}\text { Idiap database total images } \\
\text { including real and fake }=880\end{array}$ & 110 & $\begin{array}{l}\text { Full image }=665 \times 250 \text { pixels, } \\
\text { Croppped image }=565 \times 150\end{array}$ & PNG & HTER on full image $=0.00 \%$ & [130] \\
\hline W-DMD & $\begin{array}{l}\text { Idiap database total images } \\
\text { including real and fake }=880\end{array}$ & 110 & $\begin{array}{l}\text { Full image }=665 \times 250 \text { pixels, } \\
\text { Croppped image }=565 \times 150\end{array}$ & PNG & $\begin{array}{l}\text { EER on full and cropped image }=0.08 \% \text { and } \\
1.59 \%\end{array}$ & [129] \\
\hline Steerable pyramids & T-Image $=300$ & 100 & $100 \times 300$ pixels & Not reported & Average Classification Error rate (ACER) $=2.4 \%$ & [133] \\
\hline Total variation LBP & $\begin{array}{l}\text { SCUT database total images } \\
\text { including real and fake }=7200 \\
\text { Idiap database total images } \\
\text { including real and fake }=880\end{array}$ & $\begin{array}{l}100 \\
110\end{array}$ & Not reported & Not reported & $\begin{array}{l}\text { APCER, BPCER and ACER on both database } \\
\text { and also on full and cropped images }=0.00 \%\end{array}$ & [12] \\
\hline Transfer learning CNN with PCA and SVM & $\begin{array}{l}\text { ISPR database total images } \\
\text { including real and fake }=7560 \\
\text { Idiap database total images } \\
\text { including real and fake }=880\end{array}$ & $\begin{array}{c}7 \\
110\end{array}$ & Not reported & Not reported & $\begin{array}{l}\text { APCER, BPCER and ACER on both database } \\
\text { and also full and cropped image }=0.00 \%\end{array}$ & [129] \\
\hline $\begin{array}{l}\text { Transferable deep convolutional neural } \\
\text { network }\end{array}$ & $\begin{array}{l}\text { FVVPA database }=300 \text { instance } \\
\text { FVIPA database }=300 \text { instance }\end{array}$ & $\begin{array}{l}\text { Not } \\
\text { reported }\end{array}$ & Not reported & Not reported & $\begin{array}{l}\text { APCER }=0.4 \% \\
\text { BPCER }=0.00 \%\end{array}$ & [134] \\
\hline
\end{tabular}




\subsection{Impact of Deep Learning in Finger Vein Recognition}

Deep learning approaches have shown excellent development in FVR and various other research domains in terms of performance. Recent literature demonstrates that deep learning approaches have been successfully applied and enhance finger vein recognition methods. Feature extraction is one of the main steps in FVR. Deep learning approaches are robust to learn features directly from raw pixels, without the need for handcrafted descriptors, which greatly improves matching performance [122]. However, in conventional approaches handcrafted descriptors (Curvature, Gabor filter, Radon transform, Information capacity, etc.) are employed to extract features from finger vein images $[49,52,135]$. In addition, introducing deep learning to finger vein recognition can reduce the total processing time of recognition. In contrast, conventional finger vein identification requires much computation time to process different steps of finger vein recognition [127]. Personal accuracy is still a serious issue in finger vein algorithm. Conventional finger vein recognition technique needs complex preprocessing, with much effort needed to remove noise, extract and enhance the features before performing distance-based matching method. In these cases, however, a small correction can decrease recognition accuracy [100]. However, a deep learning approach does not need over-complex preprocessing and image processing. Moreover, deep learning methods are robust to noise and misalignment problems [100]. Although the dataset for finger vein recognition is small, the performance of deep learning finger vein recognition is remarkable. Deep learning finger vein identification method performance can be enhanced by employing large datasets, so there is a need for a large finger vein image dataset. Furthermore, applying deep learning methods to Presentation Attack Detection (PAD) approaches can also enhance the detection performance of presentation attack of finger vein image [134]. Moreover, template size of finger vein requires more memory space. Therefore, most of the conventional methods are proposed to reduce template size but none of them perform well enough. To resolve this, the $\mathrm{CNN}$ deep learning method with supervised discrete hashing method [124] are proposed, which perform very well to reduce the template size to 2000 bits. Furthermore, the methods speed up the matching process of finger vein technique.

\section{Discussion and Future Prospects}

In this paper, we review all the processing steps of FVR: image acquisition, preprocessing, feature extraction and matching. Moreover, in Section 4 we also discuss the performance of conventional, machine learning and deep learning algorithms in the FVR domain.

Although deep learning FVR methods are recognized as highly efficient, there are some problems which still need to be solved. The first problem is related to the first step of image preprocessing ROI extraction method of finger vein recognition methods. Yang et al. [44] used a super-pixel-based boundary detection method for ROI extraction, which is robust towards image variation, such as gray level and background noise to a limited extent. However, the experiment demonstrates that the super-pixel-based extraction method does not perfectly select the tracking point to detect the finger boundary. Moreover, in most of the ROI extraction method there is a problem of vein information loss. Hence, there is a need for a robust ROI extraction method to overcome the problem of information loss and improve the performance of finger vein recognition. Additionally, common factors also affecting the quality of the image are image blurring, non-uniform illumination, low contrast, temperature, humidity, gender, thickness of fat etc. As a result, degraded performance is still the main problem for finger vein recognition systems. Many conventional image quality evaluation, image enhancement and restoration methods have been developed to overcome these image quality problems. These methods enhance the finger vein image to some extent; however little attention has been given to factors of finger tissue (fat, tissue, muscle, water, etc.) which result in poor quality of finger vein image. This is still an open issue; an acceptable level of recognition performance has not yet been achieved. Therefore, more powerful deep learning image quality methods are still needed to deal with the quality of image. 
The second challenge is related to the image acquisition device, the quality of pixel affected by the aging of the sensor [77]. If the sensor of the device is used for a long time it will affect image quality, which finally affects the performance of the FVR system.

The third challenging problem is related to spoofing attacks, as with other biometric recognition approaches. FVR systems are also vulnerable to presentation attacks from printed vein images. As a result, presentation attack detection (PAD) is the most popular subject in finger vein recognition systems research in recent years. Newer spoof finger vein recognition methods are researched in References $[12,129,130,134]$, which greatly reduce spoofing attacks. However, finger vein presentation attack detection is still the most challenging task that needs to be addressed to improve the reliability of the finger vein biometric. In most spoof finger vein detection systems, two texture-based [12,129] and liveness-based [12] algorithms are employed. Both of these PAD algorithms face setbacks. The first concerns the liveness-based PAD method; liveness detection is an important defense against spoofing. To protect the FVR system from spoofing attack, liveness detection is now very efficient. In Reference [132], Fourier and wavelet transform are employed to detect fake finger vein images. Furthermore, Deng et al. [12] used pulse detection for liveness detection against presentation attack. Liveness detection algorithms show remarkable results in PAD methods. However, there are some deficiencies, detection was too strict, and only preliminary studies have been performed. Therefore, the liveness-based PAD method is still an open problem in PAD techniques. The second concerns the texture-based PAD algorithm. Qiu et al. [12] employ noise and blurriness which improves the discriminative power. Moreover, the texture-based PAD algorithms perform well on small databases. However, on large databases there is some scope for performance improvement. In addition, there is also a need to use more discriminative features as criteria for PAD techniques. Overall, there is a need to collect more data of real and presentation attacks to simulate all the possible cases of the PAD problem, in order to make the system resistant to the various possible presentation attack methods. Because of the above-mentioned problem, presentation attack detection is a challenging task.

The fourth challenge is related to the size of the existing finger vein database. Most of the conventional recognition methods work well on small databases, but there is need for large datasets to check the performance of the recognition methods. Furthermore, performance of deep learning approaches will be further enhanced on large-scale finger vein datasets.

The above problems have been partially solved, and finger vein recognition systems require considerable advances in techniques for the improvement of the method. As mentioned in Section 4.5, traditional finger vein recognition methods involve complex preprocessing (ROI extraction, image quality evaluation, image enhancement and normalization), handcrafted feature extraction and distance-based matching methods. Moreover, the performance of conventional FVR was also affected on a large dataset. Therefore, conventional finger vein identification approaches are found less robust and computationally expensive in finger vein identification. By contrast, introducing deep learning in preprocessing, feature extraction and matching stage of FVR can reduce the total processing time of recognition, improve the quality of images, and offer better capability to extract desirable features directly from finger vein image. Moreover, it also provides accurate matching that can significantly advance the state-of-the-art FVR methods. Additionally, performance of deep learning algorithms can be improved by incorporating a maximum number of layers to achieve much deeper networks, and these can then be tested on a large amount of images. In addition to the deeper networks, the performance of PAD methods could improve with large datasets. Clearly, it is a better choice to employ deep learning algorithm to texture and liveness-based PAD methods in order to get significant results. Although, the existing datasets of finger vein are limited, data samples to train deep models can be replicated using a similarity measure network. Finally, deep learning is recognized as a revolutionary mechanism for real-time finger vein recognition. 


\section{Conclusions}

This paper provided a comprehensive review on conventional, machine learning and deep learning-based finger vein recognition approaches. Algorithms were assessed in the key recognition steps of image acquisition, preprocessing, feature extraction and matching. In image acquisition, the light transmission method was considered to be the best for capturing the high-quality image. In image preprocessing, ROI extraction methods and image enhancement methods were reviewed. In addition, the conventional feature extraction methods were classified into four groups (i.e., vein-based method, local binary-based method, dimensionality-based method and minutiae-based method) and introduced in detail. For the matching stage, the distance-based matching methods and classifier-based matching methods were both exampled. Furthermore, we compared conventional and recently developed deep learning finger vein identification methods. However, deep learning methods showed significant improvement over traditional finger vein recognition techniques.

The paper presented the most recent research advancements in the field of FVR during the past decade, despite the challenges that need to be resolved. In particular, during image acquisition, a good image acquisition device is needed to improve the quality of finger vein image. A large-scale database is needed; in fact, this will be useful in the evaluation of all kinds of FVR techniques. Moreover, a high recognition spoof detection finger vein identification method is needed to identify spoof attacks. Furthermore, machine learning approaches play an important role in finger vein recognition. Introducing deep learning approaches into FVR has the potential to enhance the recognition performance in a broad sense.

In conclusion, the authors expect this work to be a useful starting point for new approaches, and a common ground for a wide range of benefits in the area of finger vein authentication and identification.

Author Contributions: K.S., H.L., I.Q. and J.G. collected and analyzed the corpora used in this paper; G.Y. and Y.Y. supervised the work; all authors contributed in reviewing the state of the art; K.S. wrote the paper.

Funding: This work is supported by National Science Foundation of China under Grant 61703235, 61472226, and 61573219 and the Key Research and Development Project of Shandong Province under Grant 2018GGX101032.

Acknowledgments: The authors would particularly like to thank the anonymous reviewers for their helpful suggestions.

Conflicts of Interest: The authors declare no conflict of interest.

\section{References}

1. Anil, K.J.; Arun, A.R.; Nandakumar, K. Introduction to Biometric; Springer: Berlin, Germany, 2011.

2. Mishra, K.N.; Mishra, K.N.; Agrawal, A. Veins Based Personal Identification Systems: A Review. Int. J. Intell. Syst. Appl. 2016, 10, 68. [CrossRef]

3. Qin, H.; He, X.; Yao, X.; Li, H. Finger-vein verification based on the curvature in Radon space. Expert Syst. Appl. 2017, 82, 151-161. [CrossRef]

4. Syazana-Itqan, K.; Syafeeza, A.R.; Saad, M.N.; Hamid, N.A.; Saad, W.H.M. A review of finger-vein biometrics identification approaches. Indian J. Sci. Technol. 2016, 9. [CrossRef]

5. Lu, Y.; Wu, S.; Fang, Z.; Xiong, N.; Yoon, S.; Park, D.S. Exploring finger vein based personal authentication for secure IoT. Future Gener. Comput. Syst. 2017, 77, 149-160. [CrossRef]

6. Kono, M. A new method for the identification of individuals by using of vein pattern matching of a finger. In Proceedings of the 5th Symposium on Pattern Measurement, Yamaguchi, Japan, 2000; pp. 9-12.

7. Tagkalakis, F.; Vlachakis, D.; Megalooikonomou, V.; Skodras, A. A Novel Approach to Finger Vein Authentication. In Proceedings of the IEEE 14th International Symposium on Biomedical Imaging (ISBI 2017), Melbourne, Australia, 18-21 April 2017.

8. Yang, J.; Shi, Y.; Jia, G. Finger-vein image matching based on adaptive curve transformation. Pattern Recognit. 2017, 66, 34-43. [CrossRef]

9. Miura, N.; Nagasaka, A.; Miyatake, T. Feature extraction of finger-vein patterns based on repeated line tracking and its application to personal identification. Mach. Vis. Appl. 2004, 15, 194-203. [CrossRef] 
10. Xi, X.; Yang, L.; Yin, Y. Learning discriminative binary codes for finger vein recognition. Pattern Recognit. 2017, 66, 26-33. [CrossRef]

11. Zheng, H.; Xu, Q.; Ye, Y.; Li, W. Effects of meteorological factors on finger vein recognition. In Proceedings of the 2017 IEEE International Conference on Identity, Security and Behavior Analysis (ISBA), New Delhi, India, 22-24 February 2017; pp. 1-8.

12. Ding, H. Anti-Spoofing a Finger Vascular Recognition Device with Pulse Detection. Available online: https:// pdfs.semanticscholar.org/a39a/ba4bcc8ec565b2f07ef1109311f3b56c4105.pdf (accessed on 18 August 2018).

13. Yin, Y.; Liu, L.; Sun, X. SDUMLA-HMT: A multimodal biometric database. In Proceedings of the Chinese Conference on Biometric Recognition, Beijing, China, 3-4 December 2011; Springer: Berlin/Heidelberg, Germany, 2011; pp. 260-268.

14. Qiu, X.; Kang, W.; Tian, S.; Jia, W.; Huang, Z. Finger Vein Presentation Attack Detection Using Total Variation Decomposition. IEEE Trans. Inf. Forensics Secur. 2018, 13, 465-477. [CrossRef]

15. Ton, B.T.; Veldhuis, R.N.J. A high quality finger vascular pattern dataset collected using a custom designed capturing device. In Proceedings of the 2013 International Conference on Biometrics (ICB), Madrid, Spain, 4-7 June 2013; pp. 1-5.

16. Lu, Y.; Xie, S.J.; Yoon, S.; Wang, Z.; Park, D.S. An available database for the research of finger vein recognition. In Proceedings of the 2013 6th International Conference on Image and Signal Processing (CISP), Hangzhou, China, 16-18 December 2013; Volume 1, pp. 410-415.

17. Yang, W.; Huang, X.; Zhou, F.; Liao, Q. Comparative competitive coding for personal identification by using finger vein and finger dorsal texture fusion. Inf. Sci. 2014, 268, 20-32. [CrossRef]

18. FV-USM Finger Vein Image Database. Available online: http://drfendi.com/fv_usm_datae (accessed on 18 August 2018).

19. Akintoye, K.A.; Shafry, M.R.M.; Abdullah, H. A Novel Approach for Finger Vein Pattern Enhancement using Gabor and Canny Edge Detector. Int. J. Comput. Appl. 2017, 157. [CrossRef]

20. VERA Finger Vein Data Base. 2014. Available online: https://www.idiap.ch/dataset/vera-fingervei (accessed on 18 August 2018).

21. Vanoni, M.; Tome, P.; Shafey, L.E.; Marcel, S. Cross-database evaluation using an open finger vein sensor. In Proceedings of the 2014 IEEE Workshop on Biometric Measurements and Systems for Security and Medical Applications (BIOMS), Rome, Italy, 17 October 2014; pp. 30-35.

22. Sapkale, M.; Rajbhoj, S.M. A biometric authentication system based on finger vein recognition. In Proceedings of the 2016 International Conference on Inventive Computation Technologies (ICICT), Coimbatore, India, 26-27 August 2016; Volume 3, pp. 1-4.

23. Kulkarni, S.; Raut, R.D.; Dakhole, P.K. A Novel Authentication System Based on Hidden Biometric Trait. Procedia Comput. Sci. 2016, 85, 255-262. [CrossRef]

24. Liu, Z.; Song, S. An embedded real-time finger-vein recognition system for mobile devices. IEEE Trans. Consum. Electron. 2012, 58. [CrossRef]

25. Xie, S.; Fang, L.; Wang, Z.; Ma, Z.; Li, J. Review of personal identification based on near infrared vein imaging of finger. In Proceedings of the 2017 2nd International Conference on Image, Vision and Computing (ICIVC), Chengdu, China, 2-4 June 2017; pp. 206-213.

26. Yang, L.; Yang, G.; Yin, Y.; Zhou, L. A survey of finger vein recognition. In Proceedings of the Chinese Conference on Biometric Recognition, Shenzhen, China, 28-29 October 2014; Springer: Cham, Switzerland, 2014; pp. 234-243.

27. Is the Future of Digital Security in Our Veins? Available online: https://motherboard.vice.com/en_us/ article/wnjzen/future-digital-security-is-in-our-veins (accessed on 18 August 2018).

28. Retail Systems. Available online: www.retailsystemspl.com.au (accessed on 18 August 2018).

29. Wang, K.Q.; Khisa, A.S.; Wu, X.Q.; Zhao, Q.S. Finger vein recognition using LBP variance with global matching. In Proceedings of the Wavelet Analysis and Pattern Recognition (ICWAPR), 2012 International Conference, Guangdong, China, 2012; pp. 196-201.

30. Ma, H.; Wang, K.; Fan, L.; Cui, F. A finger vein image quality assessment method using object and human visual system index. In Proceedings of the International Conference on Intelligent Science and Intelligent Data Engineering, Nanjing, China, 15-17 October 2012; Springer: Berlin/Heidelberg, Germany, 2012; pp. 498-506. 
31. Qin, H.; Li, S.; Kot, A.C.; Qin, L. Quality assessment of finger-vein image. In Proceedings of the 2012 Asia Pacific Signal \& Information Processing Association Annual Summit and Conference (APSIPA ASC), Hollywood, CA, USA, 3-6 December 2012; pp. 1-4.

32. Xie, S.J.; Zhou, B.; Yang, J.; Lu, Y.; Pan, Y. Novel hierarchical structure based finger vein image quality assessment. In Biometric Recognition; Springer: Cham, Switzerland, 2013; pp. 266-273.

33. Nguyen, D.T.; Park, Y.H.; Shin, K.Y.; Park, K.R. New Finger-vein Recognition Method Based on Image Quality Assessment. KSII Trans. Internet Inf. Syst. 2013, 7. [CrossRef]

34. Peng, J.; Li, Q.; Niu, X. A novel finger vein image quality evaluation method based on triangular norm. In Proceedings of the Tenth International Conference on Intelligent Information Hiding and Multimedia Signal Processing (IIH-MSP), Kitakyushu, Japan, 27-29 August 2014; pp. 239-242.

35. Yang, L.; Yang, G.; Yin, Y.; Xiao, R. Finger vein image quality evaluation using support vector machines. Opt. Eng. 2013, 52, 027003. [CrossRef]

36. Zhou, L.; Yang, G.; Yang, L.; Yin, Y.; Li, Y. Finger vein image quality evaluation based on support vector regression. Int. J. Signal Process. Image Process. Pattern Recognit. 2015, 8, 211-222. [CrossRef]

37. Qin, H.; El-Yacoubi, M.A. Finger-vein quality assessment by representation learning from binary images. In Proceedings of the International Conference on Neural Information Processing, Istanbul, Turkey, 9-12 November 2015; Springer: Cham, Switzerland, 2015; pp. 421-431.

38. Huang, Z.; Kang, W.; Wu, Q.; Zhao, J.; Jia, W. A Finger Vein Identification System Based on Image Quality Assessment. In Proceedings of the Chinese Conference on Biometric Recognition, Chengdu, China, 14-16 October 2016; Springer: Cham, Switzerland, 2016; pp. 244-254.

39. Brindha, S. Finger Vein Recognition. Int. J. Renew. Energy Technol. 2017, 4, 1298-1300.

40. Hoshyar, A.N.; Sulaiman, R. Review on finger vein authentication system by applying neural network. In Proceedings of the 2010 International Symposium on Information Technology (ITSim), Kuala Lumpur, Malaysia, 15-17 June 2010; Volume 2, pp. 1020-1023.

41. You, L.; Li, H.; Wang, J. Finger-vein recognition algorithm based on potential energy theory. In Proceedings of the 2015 IEEE 16th International Conference on Communication Technology (ICCT), Hangzhou, China, 18-20 October 2015; pp. 678-683.

42. Yang, J.; Shi, Y. Finger-vein ROI localization and vein ridge enhancement. Pattern Recognit. Lett. 2012, 33, 1569-1579. [CrossRef]

43. Podgantwar, U.D.; Raut, U.K. Extraction of finger vein patterns using gabor filter in finger vein image profiles. Int. J. Eng. Res. Technol. 2013, 2, 3294-3298.

44. Lu, Y.; Yang, G.; Zhou, L.; Yin, Y. Superpixel based finger vein roi extraction with sensor interoperability. In Proceedings of the 2015 International Conference on Biometrics (ICB), Phuket, Thailand, 19-22 May 2015; pp. 444-451.

45. Wang, M.; Tang, D. Region of interest extraction for finger vein images with less information losses. Multimed. Tools Appl. 2017, 76, 14937-14949. [CrossRef]

46. Zheng, H.; Ye, Y.; Liu, S.; Ni, N.; Zhang, Y.; Huang, H.; Li, W. Parameter adjustment of finger vein recognition algorithms. In Proceedings of the Identity, Security and Behavior Analysis (ISBA), 2017 IEEE International Conference, New Delhi, India, 2017; pp. 1-8.

47. Ezhilmaran, D.; Joseph, P.R.P. A Study of Feature Extraction Techniques and Image Enhancement Algorithms for Finger Vein Recognition. Int. J. PharmTech Res. 2015, 8, 222-229.

48. Xie, S.J.; Lu, Y.; Yoon, S.; Yang, J.; Park, D.S. Intensity variation normalization for finger vein recognition using a guided filter based singe scale retinex. Sensors 2015, 15, 17089-17105. [CrossRef] [PubMed]

49. Kauba, C.; Reissig, J.; Uhl, A. Pre-processing cascades and fusion in finger vein recognition. In Proceedings of the 2014 International Conference of the Biometrics Special Interest Group (BIOSIG), Darmstadt, Germany, 10-12 September 2014; pp. 1-6.

50. Raghavendra, R.; Busch, C. Exploring dorsal finger vein pattern for robust person recognition. In Proceedings of the 2015 International Conference on Biometrics (ICB), Phuket, Thailand, 19-22 May 2015; pp. 341-348.

51. Mohan, M.; Kumar, R.P.; Agrawal, R.; Sharma, S.; Dutta, M.K.; Travieso, C.M.; Alonso-Hernandez, J.B. Finger vein recognition using Integrated Responses of Texture features. In Proceedings of the 20154 th International Work Conference on Bioinspired Intelligence (IWOBI), San Sebastian, Spain, 10-12 June 2015; pp. 209-214. 
52. Yang, J.; Shi, Y.; Yang, J.; Jiang, L. A novel finger-vein recognition method with feature combination. In Proceedings of the 2009 16th IEEE International Conference on Image Processing (ICIP), Cairo, Egypt, 7-10 November 2009; pp. 2709-2712.

53. Chen, L.; Wang, J.; Yang, S.; He, H. A Finger Vein Image-Based Personal Identification System with Self-Adaptive Illuminance Control. IEEE Trans. Instrum. Meas. 2017, 66, 294-304. [CrossRef]

54. Jing, Z.; Yang, J. Finger-vein image enhancement based on combination of gray-level grouping and circular gabor filter. In Proceedings of the 2009 International Conference on Information Engineering and Computer Science (ICIECS 2009), Wuhan, China, 19-20 December 2009; pp. 1-4.

55. Pflug, A.; Hartung, D.; Busch, C. Feature extraction from vein images using spatial information and chain codes. Inf. Secur. Tech. Rep. 2012, 17, 26-35. [CrossRef]

56. Yang, J.; Yang, J. Multi-channel gabor filter design for finger-vein image enhancement. In Proceedings of the 2009 Fifth International Conference on Image and Graphics (ICIG'09), Xi'an, China, 20-23 September 2009; pp. 87-91.

57. Yang, J.; Shi, Y.; Yang, J. Finger-vein recognition based on a bank of Gabor filters. In Proceedings of the Asian Conference on Computer Vision, Xi'an, China, 23-27 September 2009; Springer: Berlin/Heidelberg, Germany, 2009; pp. 374-383.

58. Wang, K.; Liu, J.; Oluwatoyin, P.P.; Feng, W. Finger vein identification based on 2-D gabor filter. In Proceedings of the 2010 2nd International Conference on Industrial Mechatronics and Automation (ICIMA), Wuhan, China, 30-31 May 2010; Volume 2, pp. 10-13.

59. Xie, S.J.; Yang, J.C.; Yoon, S.; Yu, L.; Park, D.S. Guided Gabor filter for finger vein pattern extraction. In Proceedings of the 2012 Eighth International Conference on Signal Image Technology and Internet Based Systems (SITIS), Naples, Italy, 25-29 November 2012; pp. 118-123.

60. Yang, J.; Li, X. Efficient finger vein localization and recognition. In Proceedings of the 2010 20th International Conference on Pattern Recognition (ICPR), Istanbul, Turkey, 23-26 August 2010; pp. 1148-1151.

61. Khellat-kihel, S.; Abrishambaf, R.; Cardoso, N.; Monteiro, J.; Benyettou, M. Finger vein recognition using Gabor filter and Support Vector Machine. In Proceedings of the Image Processing, Applications and Systems Conference (IPAS), Sfax, Tunisia, 5-7 November 2014; pp. 1-6.

62. Liu, T.; Xie, J.B.; Yan, W.; Li, P.Q.; Lu, H.Z. An algorithm for finger-vein segmentation based on modified repeated line tracking. Imaging Sci. J. 2013, 61, 491-502. [CrossRef]

63. Kaur, P.; Verma, P. Human Identification with Finger Veins Using Repeated Line Tracking, Even Gabor and Automatic Trimap Generation Algorithms. Int. J. Comput. Sci. Inf. Technol. 2014, 5, 8003-8007.

64. Kaur, M.; Babbar, G. Finger Vein Detection using Repeated Line Tracking, Even Gabor and Multilinear Discriminant Analysis (MDA). Int. J. Comput. Sci. Inf. Technol. 2015, 6, 3280-3284.

65. Veluchamy, S.; Karlmarx, L.R. System for multimodal biometric recognition based on finger knuckle and finger vein using feature-level fusion and k-support vector machine classifier. IET Biom. 2016, 6, 232-242. [CrossRef]

66. Miura, N.; Nagasaka, A.; Miyatake, T. Extraction of finger-vein patterns using maximum curvature points in image profiles. IEICE Trans. Inf. Syst. 2007, 90, 1185-1194. [CrossRef]

67. Tagkalakis, F.; Fotopoulos, V. A low cost finger vein authentication system, using maximum curvature points. In Proceedings of the 2015 International Conference on Applied Electronics (AE), Pilsen, Czech Republic, 8-9 September 2015; pp. 249-252.

68. Song, W.; Kim, T.; Kim, H.C.; Choi, J.H.; Kong, H.J.; Lee, S.R. A finger-vein verification system using mean curvature. Pattern Recognit. Lett. 2011, 32, 1541-1547. [CrossRef]

69. Choi, J.H.; Song, W.; Kim, T.; Lee, S.R.; Kim, H.C. Finger vein extraction using gradient normalization and principal curvature. In Proceedings of the SPIE-IS\&T Electronic Imaging, San Jose, CA, USA, 18-22 January 2009.

70. Yahaya, H.Y.; Shamshudin, M.S.; Leng, Y.W. Finger vein feature extraction using discretization. In Proceeding of the 4th International Conference on Artificial Intelligence and Computer Science (AICS2016), Langkawi, Malaysia, 28-29 November 2016.

71. Lee, H.C.; Kang, B.J.; Lee, E.C.; Park, K.R. Finger vein recognition using weighted local binary pattern code based on a support vector machine. J. Zhejiang Univ. Sci. C 2010, 11, 514-524. [CrossRef]

72. Lee, E.C.; Lee, H.C.; Park, K.R. Finger vein recognition using minutia-based alignment and local binary pattern-based feature extraction. Int. J. Imaging Syst. Technol. 2009, 19, 179-186. [CrossRef] 
73. Lee, E.C.; Jung, H.; Kim, D. New finger biometric method using near infrared imaging. Sensors 2011, 11, 2319-2333. [CrossRef] [PubMed]

74. Rosdi, B.A.; Shing, C.W.; Suandi, S.A. Finger vein recognition using local line binary pattern. Sensors 2011, 11, 11357-11371. [CrossRef] [PubMed]

75. Zhang, B.; Gao, Y.; Zhao, S.; Liu, J. Local derivative pattern versus local binary pattern: Face recognition with high-order local pattern descriptor. IEEE Trans. Image Process. 2010, 19, 533-544. [CrossRef] [PubMed]

76. Lu, Y.; Xie, S.J.; Yoon, S.; Park, D.S. Finger vein identification using polydirectional local line binary pattern. In Proceedings of the 2013 International Conference on ICT Convergence (ICTC), Jeju Island, Korea, 14-16 October 2013; pp. 61-65.

77. Lu, Y.; Yoon, S.; Xie, S.J.; Yang, J.; Wang, Z.; Park, D.S. Finger Vein Recognition Using Generalized Local Line Binary Pattern. KSII Trans. Internet Inf. Syst. 2014, 8. [CrossRef]

78. Liu, H.; Song, L.; Yang, G.; Yang, L.; Yin, Y. Customized Local Line Binary Pattern Method for Finger Vein Recognition. In Proceedings of the Chinese Conference on Biometric Recognition, Beijing, China, 28-29 October 2017; Springer: Cham, Switzerland; pp. 314-323.

79. Xi, X.; Yang, G.; Yin, Y.; Meng, X. Finger vein recognition with personalized feature selection. Sensors 2013, 13, 11243-11259. [CrossRef] [PubMed]

80. Dong, L.; Yang, G.; Yin, Y.; Liu, F.; Xi, X. Finger vein verification based on a personalized best patches map. In Proceedings of the 2014 IEEE International Joint Conference on Biometrics (IJCB), Clearwater, FL, USA, 29 September-2 October 2014; pp. 1-8.

81. Al-Nima, R.R.O.; Abdullah, M.A.M.; Al-Kaltakchi, M.T.S.; Dlay, S.S.; Woo, W.L.; Chambers, J.A. Finger texture biometric verification exploiting multi-scale sobel angles local binary pattern features and score-based fusion. Digit. Signal Process. 2017, 70, 178-189. [CrossRef]

82. Yang, G.; Xiao, R.; Yin, Y.; Yang, L. Finger vein recognition based on personalized weight maps. Sensors 2013, 13, 12093-12112. [CrossRef] [PubMed]

83. Liu, Z.; Yin, Y.; Wang, H.; Song, S.; Li, Q. Finger vein recognition with manifold learning. J. Netw. Comput. Appl. 2010, 33, 275-282. [CrossRef]

84. Guan, F.; Wang, K.; Wu, Q. Bi-directional weighted modular b2dpca for finger vein recognition. In Proceedings of the 2010 3rd International Conference on Image and Signal Processing (CISP), Yantai, China, 16-18 October 2010; Volume 1, pp. 93-97.

85. Wu, J.D.; Liu, C.T. Finger-vein pattern identification using principal component analysis and the neural network technique. Expert Syst. Appl. 2011, 38, 5423-5427. [CrossRef]

86. Wu, J.D.; Liu, C.T. Finger-vein pattern identification using SVM and neural network technique. Expert Syst. Appl. 2011, 38, 14284-14289. [CrossRef]

87. Yang, G.; Xi, X.; Yin, Y. Finger vein recognition based on (2D) 2 PCA and metric learning. J. Biomed. Biotechnol. 2012, 2012, 324249. [CrossRef] [PubMed]

88. Hajian, A.; Damavandinejadmonfared, S. Optimal feature extraction dimension in finger vein recognition using kernel principal component analysis. Int. J. Comput. Inf. Syst. Control Eng. 2014, 8, 1637-1640.

89. You, L.; Wang, J.; Li, H.; Li, X. Finger Vein Recognition Based on 2DPCA and KMMC. Int. J. Signal Process. Image Process. Pattern Recognit. 2015, 8, 163-170. [CrossRef]

90. Vlachos, M.; Dermatas, E. Finger vein segmentation from infrared images based on a modified separable mumford shah model and local entropy thresholding. Comput. Math. Methods Med. 2015, 2015, 868493. [CrossRef] [PubMed]

91. Li, X.; Wan, M.K. A Face Recognition System Based on Kernel Maximum Between-class Margin Criterion (KMMC). J. Nanchang Hangkong Univ. 2013, 3, 6.

92. Minutiae Based Fingerprint. Available online: https://www.bayometric.com/minutiae-based-extractionfingerprint-recognition/ (accessed on 18 August 2018).

93. Zafar, W.; Ahmad, T.; Hassan, M. Minutiae based fingerprint matching techniques. In Proceedings of the 2014 IEEE 17th International Multi-Topic Conference (INMIC), Karachi, Pakistan, 8-10 December 2014; pp. 411-416.

94. Zaeri, N. Minutiae-based fingerprint extraction and recognition. In Biometrics; InTech: London, UK, 2011.

95. Chen, W.; Gao, Y. A minutiae-based fingerprint matching algorithm using phase correlation. In Proceedings of the 9th Biennial Conference of the Australian Pattern Recognition Society on Digital Image Computing Techniques and Applications, Glenelg, Australia, 3-5 December 2007; pp. 233-238. 
96. Mantrao, N.; Sukhpreet, K. An Efficient Minutiae Matching Method for Finger Vein Recognition. Int. J. Adv. Res. Comput. Sci. Softw. Eng. 2015, 5, 657-660.

97. Aziz, W.N.; Abdullah, I.; Seman, K.; Sayuti, N.N.S. Finger vein minutiae points extraction based on maximum curvature points in image profile and fingerprint application methods. Aust. J. Basic Appl. Sci. 2013, 7, 751-756.

98. Prabhakar, P.; Thomas, T. Finger vein identification based on minutiae feature extraction with spurious minutiae removal. In Proceedings of the 2013 Third International Conference on Advances in Computing and Communications (ICACC), Kerala, India, 29-31 August 2013; pp. 196-199.

99. Liu, C.; Kim, Y.H. An efficient finger-vein extraction algorithm based on random forest regression with efficient local binary patterns. In Proceedings of the 2016 IEEE International Conference on Image Processing (ICIP), Phoenix, AZ, USA, 25-28 September 2016; pp. 3141-3145.

100. Radzi, S.A.; Hani, M.K.; Bakhteri, R. Finger-vein biometric identification using convolutional neural network. Turk. J. Electr. Eng. Comput. Sci. 2016, 24, 1863-1878. [CrossRef]

101. Guan, F.; Wang, K.; Yang, Q. A study of two direction weighted (2D) 2 LDA for finger vein recognition. In Proceedings of the 2011 4th International Congress on Image and Signal Processing (CISP), Shanghai, China, 15-17 October 2011; Volume 2, pp. 860-864.

102. Yang, W.; Rao, Q.; Liao, Q. Personal identification for single sample using finger vein location and direction coding. In Proceedings of the 2011 International Conference on Hand-Based Biometrics (ICHB), Hongkong, China, 17-18 November 2011; pp. 1-6.

103. Meng, X.; Yang, G.; Yin, T.; Xiao, R. Finger vein recognition based on local directional code. Sensors 2012, 12, 14937-14952. [CrossRef] [PubMed]

104. Yang, G.; Xi, X.; Yin, Y. Finger vein recognition based on a personalized best bit map. Sensors 2012, 12, 1738-1757. [CrossRef] [PubMed]

105. Harsha, P.; Subashini, C. A real time embedded novel finger-vein recognition system for authenticated on teller machine. In Proceedings of the 2012 International Conference on Emerging Trends in Electrical Engineering and Energy Management (ICETEEEM), Chennai, India, 13-15 December 2012; pp. 271-275.

106. Damavandinejadmonfared, S. Finger vein recognition using linear kernel entropy component analysis. In Proceedings of the 2012 IEEE International Conference on Intelligent Computer Communication and Processing (ICCP), Cluuj, Romania, 30 August-1 September 2012; pp. 249-252.

107. Yang, J.; Shi, Y. Towards finger-vein image restoration and enhancement for finger-vein recognition. Inf. Sci. 2014, 268, 33-52. [CrossRef]

108. Vega, A.P.; Travieso, C.M.; Alonso, J.B. Biometric personal identification system based on patterns created by finger veins. In Proceedings of the 2014 International Work Conference on Bio-inspired Intelligence (IWOBI), Liberia, Costa Rica, 16-18 July 2014; pp. 65-70.

109. Lu, Y.; Yoon, S.; Xie, S.J.; Yang, J.; Wang, Z.; Park, D.S. Finger vein recognition using histogram of competitive gabor responses. In Proceedings of the 2014 22nd International Conference on Pattern Recognition (ICPR), Stockholm, Sweden, 24-28 August 2014; pp. 1758-1763.

110. Liu, X.Z.; Yang, G. Block-wise two-dimensional maximum margin criterion for face recognition. Sci. World J. 2014. [CrossRef] [PubMed]

111. Gupta, P.; Gupta, P. An accurate finger vein based verification system. Digit. Signal Process. 2015, 38, 43-52. [CrossRef]

112. Liu, B.C.; Xie, S.J.; Park, D.S. Finger vein recognition using optimal partitioning uniform rotation invariant LBP descriptor. J. Electr. Comput. Eng. 2016. [CrossRef]

113. Hoshyar, A.N.; Sulaiman, R. Smart access control with finger vein authentication and neural network. J. Am. Sci. 2011, 7, 192-200.

114. Malik, I.; Sharma, R. Analysis of different techniques for finger-vein feature extraction. Int. J. Comput. Trends Technol. 2013, 4, 1301-1305.

115. Xie, S.S.; Yoon, S.; Yang, J.; Lu, Y.; Park, D.S.; Zhou, B. Feature component-based extreme learning machines for finger vein recognition. Cogn. Comput. 2014, 6, 446-461. [CrossRef]

116. Kumar, A.; Zhou, Y. Human identification using finger images. IEEE Trans. Image Process. 2012, 21, $2228-2244$. [CrossRef] [PubMed]

117. Zhou, L.; Yang, G.; Yin, Y.; Yang, L.; Wang, K. Finger Vein Recognition Based on Stable and Discriminative Superpixels. Int. J. Pattern Recognit. Artif. Intell. 2016, 30. [CrossRef] 
118. Wang, G.; Wang, J. SIFT Based Vein Recognition Models: Analysis and Improvement. Comput. Math. Methods Med. 2017. [CrossRef] [PubMed]

119. Mobarakeh, A.K.; Rizi, S.M.; Khaniabadi, S.M.; Bagheri, M.L.; Nazari, S. Applying Weighted K-nearest centroid neighbor as classifier to improve the finger vein recognition performance. In Proceedings of the 2012 IEEE International Conference on Control System, Computing and Engineering (ICCSCE), Penang, Malaysia, 23-25 November 2012; pp. 56-59.

120. William, A.; Ong, T.S.; Tee, C.; Goh, M.K.O. Multi-instance finger vein recognition using local hybrid binary gradient contour. In Proceedings of the 2015 Asia-Pacific Signal and Information Processing Association Annual Summit and Conference (APSIPA), Hong Kong, China, 16-19 December 2015; pp. 1226-1231.

121. Qin, H.; El-Yacoubi, M.A. Deep Representatn-Based Feature Extraction and Recovering for Finger-Vein Verification. IEEE Trans. Inf. Forensics Secur. 2017, 12, 1816-1829. [CrossRef]

122. Qin, H.; El Yacoubi, A.M. Deep Representation for Finger-vein Image Quality Assessment. IEEE Trans. Circuits Syst. Video Technol. 2018, 28, 1677-1693. [CrossRef]

123. Huang, H.; Liu, S.; Zheng, H.; Ni, L.; Zhang, Y.; Li, W. DeepVein: Novel finger vein verification methods based on Deep Convolutional Neural Networks. In Proceedings of the 2017 IEEE International Conference on Identity, Security and Behavior Analysis (ISBA), New Delhi, India, 22-24 February 2017; pp. 1-8.

124. Xie, C.; Kumar, A. Finger Vein Identification Using Convolutional Neural Network and Supervised Discrete Hashing. In Deep Learning for Biometrics; Springer: Cham, Switzerland, 2017; pp. 109-132.

125. Liu, Y.; Ling, J.; Liu, Z.; Shen, J.; Gao, C. Finger vein secure biometric template generation based on deep learning. Soft Comput. 2017, 22, 2257-2265. [CrossRef]

126. Hong, H.G.; Lee, M.B.; Park, K.R. Convolutional Neural Network-Based Finger-Vein Recognition Using NIR Image Sensors. Sensors 2017, 17, 1297. [CrossRef] [PubMed]

127. Meng, G.; Fang, P.; Zhang, B. Finger vein recognition based on convolutional neural network. In Proceedings of the MATEC Web of Conferences, Zhuhai, China, 23-24 September 2017; EDP Sciences: Les Ulis, France, 2017; Volume 128, p. 04015.

128. Fang, Y.; Wu, Q.; Kang, W. A novel finger vein verification system based on two-stream convolutional network learning. Neurocomputing 2018. [CrossRef]

129. Raghavendra, R.; Busch, C. Presentation attack detection algorithms for finger vein biometrics: A comprehensive study. In Proceedings of the 2015 11th International Conference on Signal-Image Technology \& Internet-Based Systems (SITIS), Bangkok, Thailand, 23-27 November 2015; pp. 628-632.

130. Tome, P.; Raghavendra, R.; Busch, C.; Tirunagari, S.; Poh, N.; Shekar, B.H.; Gragnaniello, D.; Sansone, C.; Verdoliva, L.; Marcel, S. The 1st competition on counter measures to finger vein spoofing attacks. In Proceedings of the 2015 International Conference on Biometrics (ICB), Phuket, Thailand, 19-22 May 2015; pp. 513-518.

131. Nguyen, D.T.; Yoon, H.S.; Pham, T.D.; Park, K.R. Spoof Detection for Finger-Vein Recognition System Using NIR Camera. Sensors 2017, 17, 2261. [CrossRef] [PubMed]

132. Nguyen, D.T.; Park, Y.H.; Shin, K.Y.; Kwon, S.Y.; Lee, H.C.; Park, K.R. Fake finger-vein image detection based on fourier and wavelet transforms. Digit. Signal Process. 2013, 23, 1401-1413. [CrossRef]

133. Tirunagari, S.; Poh, N.; Bober, M.; Windridge, D. Windowed DMD as a microtexture descriptor for finger vein counter-spoofing in biometrics. In Proceedings of the IEEE International Workshop on Information Forensics and Security (WIFS), Rome, Italy, 16-19 November 2015; pp. 1-6.

134. Raghavendra, R.; Venkatesh, S.; Raja, K.B.; Busch, C. Transferable deep convolutional neural network features for fingervein presentation attack detection. In Proceedings of the 2017 5th International Workshop on Biometrics and Forensics (IWBF), Conventry, UK, 4-5 April 2017; pp. 1-5.

135. Cho, S.R.; Park, Y.H.; Nam, G.P.; Shin, K.Y.; Lee, H.C.; Park, K.R.; Kim, S.M.; Kim, H.C. Enhancement of finger-vein image by vein line tracking and adaptive gabor filtering for finger-vein recognition. In Applied Mechanics and Materials; Trans Tech Publications: Zürich, Switzerland, 2012; Volume 145, pp. 219-223.

(C) 2018 by the authors. Licensee MDPI, Basel, Switzerland. This article is an open access article distributed under the terms and conditions of the Creative Commons Attribution (CC BY) license (http:/ / creativecommons.org/licenses/by/4.0/). 